\title{
COMPACT COMPLEX SURFACES ADMITTING NON-TRIVIAL SURJECTIVE ENDOMORPHISMS
}

\author{
YOSHIO FUJIMOTO* AND NOBORU NAKAYAMA
}

\begin{abstract}
Smooth compact complex surfaces admitting non-trivial surjective endomorphisms are classified up to isomorphisms. The algebraic case has been classified in [3], [19]. The following surfaces are listed in the non-algebraic case: a complex torus, a Kodaira surface, a Hopf surface with at least two curves, an Inoue surface with curves, and an Inoue surface without curves satisfying a rationality condition.
\end{abstract}

\section{INTRODUCTION}

A non-trivial surjective endomorphism of a compact complex variety $X$ is a nonisomorphic surjective morphism $X \rightarrow X$ by definition. Projective surfaces $X$ admitting non-trivial surjective endomorphisms are classified in [3], [19] as follows:

(1) $X$ is a toric surface;

(2) $X$ is a $\mathbb{P}^{1}$-bundle over an elliptic curve;

(3) $X$ is a $\mathbb{P}^{1}$-bundle over a non-singular curve $C$ of genus $g \geq 2$ such that $X \times_{C} C^{\prime} \simeq$ $\mathbb{P}^{1} \times C^{\prime}$ for a finite étale covering $C^{\prime} \rightarrow C$;

(4) $X$ is an abelian surface or a hyperelliptic surface;

(5) $X$ is an elliptic surface with the Kodaira dimension $\kappa(X)=1$ and the topological Euler number $e(X)=0$.

The cases above correspond to the following numerical invariants: (1) $\kappa(X)=-\infty$ and the irregularity $q(X)=0 ;(2) \kappa(X)=-\infty$ and $q(X)=1 ;(3) \kappa(X)=-\infty$ and $q(X) \geq 2$; (4) $\kappa(X)=0 ;(5) \kappa(X)=1$. Note that a surface of general type does not admit non-trivial surjective endomorphisms. In this article, we study the case where $X$ is non-algebraic. The following is our main result:

THEOREM 1.1. The non-algebraic non-singular compact complex surfaces $X$ admitting non-trivial surjective endomorphisms are classified as follows:

(1) $X$ is a complex torus;

(2) $X$ is a primary Kodaira surface, a secondary Kodaira surface, or an elliptic Hopf surface;

2000 Mathematics Subject Classification. 14J25, 14J27, 32J15.

Key words and phrases. endomorphism, elliptic surface, non-algebraic surface, $\mathrm{VII}_{0}$ surface, Kodaira surface, Inoue surface.

*Partially supported by the Sumitomo Foundation. 
(3) $X$ is a Hopf surface with two elliptic curves or one of the following Inoue surfaces without curves: $S_{M}, S_{N, p, q, r ; t}^{(+)}$satisfying a rationality condition (cf. Theorem 8.6) on the parameter $t$, and $S_{N, p, q, r}^{(-)}$;

(4) $X$ is a successive blowups of one of the following surfaces whose centers are nodes of curves: a parabolic Inoue surface, a hyperbolic Inoue surface, and a half Inoue surface.

The cases above correspond to the following numerical invariants: (1) the first Betti number $b_{1}(X)$ is even; (2) $b_{1}(X)$ is odd and the algebraic dimension $a(X)=1$; (3) $a(X)=0, b_{1}(X)=1$, and $b_{2}(X)=0 ;(4) a(X)=0, b_{1}(X)=1$, and $b_{2}(X)>0$. In particular, if $X$ is Kähler, then $X$ is a complex torus. The definitions of Kodaira surfaces, Hopf surfaces, Inoue surfaces are given in [10], [5], [7] (cf. [1]). But we discuss the structures and the properties of these non-Kähler surfaces in Sections 2, 6-9 below. The Kodaira surfaces $X$ are characterized by the conditions: $b_{1}(X)$ is odd and $c_{1}(X)=0$ in $H^{2}(X, \mathbb{Q})$. A Hopf surface is a compact complex surface whose universal covering space is biholomorphic to $\mathbb{C}^{2} \backslash\{(0,0)\}$ by definition. A compact complex surface is called a surface of class VII if the first Betti number is 1 . If it is minimal, furthermore, it is called a surface of class $\mathrm{VII}_{0}$. Hopf surfaces and Inoue surfaces are typical examples of surfaces of class $\mathrm{VII}_{0}$ with the algebraic dimension zero.

The idea of the proof of Theorem 1.1 is as follows: In the first step, we list the possible surfaces $X$ admitting a non-trivial surjective endomorphism. We can show that, for such an $X$, the set $\mathcal{S}(X)$ of curves with negative self-intersection number is finite by the same argument as in [19]. This yields a strong condition on $X$. For example, it implies that if $X$ is a non-algebraic elliptic surface, equivalently if $a(X)=1$, then the singular fibers are multiple of elliptic curves (cf. Proposition 4.1). Furthermore by investigating the variation of Hodge structure, we infer that $X$ is one of the surfaces listed in (2) of Theorem 1.1 (cf. Theorem 4.5). The finiteness of $\mathcal{S}(X)$ and some known results on surfaces of class $\mathrm{VII}_{0}$ imply that if $X$ is a surface of class VII, then its minimal model is one of the known examples (cf. Theorem 5.2). Thus we can make a list of the possible surfaces.

Conversely in the second step, we examine whether a non-trivial surjective endomorphism exists or not individually for the cases of surfaces listed as candidates. It seems to be difficult to determine the existence on Kodaira surfaces, on non-elliptic Hopf surfaces, and on Inoue surfaces without curves, because of their complicated construction from the universal covering space. We consider a lift of an expected endomorphism to the universal covering space and examine whether it really induces a non-trivial endomorphism by elementary and long calculations. In the case of Kodaira surfaces and Inoue surfaces without curves, we can describe the induced endomorphism of the fundamental group explicitly by using triangular matrices in GL(3, $\mathbb{C}$ ) (cf. Proposition 6.4, Proposition 8.5). Our method is delicate but powerful enough for the investigation. For example, we find a remarkable condition on the parameter $t$ for the existence of endomorphism on the Inoue 
surface $S_{N, p, q, r ; t}^{(+)}$. Contrary to the above, in the case of elliptic Hopf surfaces, we look at the behavior of multiple fibers of the elliptic fibration. If it has three multiple fibers, then it is obtained as the quotient of an elliptic fiber bundle over $\mathbb{P}^{1}$ by a free action of a regular polyhedral group $G \subset \operatorname{PGL}(2, \mathbb{C})$. A $G$-equivariant endomorphism on the elliptic bundle is constructed by a similar method as in Lemma 6 in [19].

This paper is organized as follows: After explaining the classification theory of nonalgebraic surfaces in Section 2, we recall and generalize the argument in [19] on the set $\mathcal{S}(X)$ of curves with negative self-intersection numbers in Section 3. The possible surfaces $X$ are listed in Section 4 and Section 5, respectively for the cases $a(X)=1$ and $a(X)=0$. The existence of endomorphisms is studied individually for the cases of surfaces in Sections 6, 7, 8, and 9 according to Kodaira surfaces, Hopf surfaces, Inoue surfaces without curves, and Inoue surfaces with curves.

\section{NotATiON}

Throughout this paper, we call a compact complex analytic surface by a surface and a compact complex analytic curve by a curve, for short, if it causes no confusion.

Let $X$ be a non-singular compact complex surface. For $u \in \mathrm{H}^{i}(X, \mathbb{Z}), v \in \mathrm{H}^{4-i}(X, \mathbb{Z})$, we denote by $u \cdot v$ the intersection number $\int u \cup v$, where $\cup$ is the cup-product and $\int$ is the trace map $\mathrm{H}^{4}(X, \mathbb{Z}) \rightarrow \mathbb{Z}$. A divisor $D$ of $X$ defines a homology class in $\mathrm{H}_{2}(X, \mathbb{Z})$ which corresponds to the first Chern class $c_{1}(D)=c_{1}\left(\mathcal{O}_{X}(D)\right)$ associated with the line bundle $\mathcal{O}_{X}(D)$ by the Poincaré isomorphism $\mathrm{H}^{2}(X, \mathbb{Z}) \simeq \mathrm{H}_{2}(X, \mathbb{Z})$. The intersection number $c_{1}\left(D_{1}\right) \cdot c_{1}\left(D_{2}\right)$ of two divisors $D_{1}$ and $D_{2}$ is denoted by $D_{1} \cdot D_{2}$. Note that $c_{1}(\mathcal{L}) \cdot C=\left.\operatorname{deg} \mathcal{L}\right|_{C}$ for a line bundle $\mathcal{L}$ and for an irreducible curve $C$.

Let $f: Y \rightarrow X$ be a surjective morphism from another non-singular compact complex surface. It induces the pull-back $f^{*}: \mathrm{H}^{i}(X, \mathbb{Z}) \rightarrow \mathrm{H}^{i}(Y, \mathbb{Z})$ and the push-forward $f_{*}: \mathrm{H}_{i}(Y, \mathbb{Z}) \rightarrow \mathrm{H}_{i}(X, \mathbb{Z})$. By the Poincaré duality, the push-forward induces a homomorphism $\mathrm{H}^{i}(Y, \mathbb{Z}) \rightarrow \mathrm{H}^{i}(X, \mathbb{Z})$, which we also denote by $f_{*}$. Then the composite $f_{*} \circ f^{*}: \mathrm{H}^{i}(X, \mathbb{Z}) \rightarrow \mathrm{H}^{i}(X, \mathbb{Z})$ is the multiplication map by $\operatorname{deg} f$ : the mapping degree of $f$. The projection formula $f_{*}\left(f^{*} x \cdot y\right)=x \cdot f_{*} y$ holds for $x \in \mathrm{H}^{i}(X, \mathbb{Z})$ and $y \in \mathrm{H}^{4-i}(Y, \mathbb{Z})$. For a divisor $D$ on $X$ and a divisor $E$ on $Y$, we have $c_{1}\left(f^{*} D\right)=f^{*} c_{1}(D)$ and $c_{1}\left(f_{*} E\right)=f_{*} c_{1}(E)$, where $f^{*} D$ and $f_{*} E$ are the pull-back and the push-forward as divisors, respectively.

Contrary to the case of algebraic surfaces, the canonical line bundle $\omega_{X}=\Omega_{X}^{2}$ may not have a non-zero global meromorphic section. The divisor of the meromorphic section is called canonical and is denoted by $K_{X}$. Even if the canonical divisor does not exist, we use the same symbol $K_{X}$ as the canonical divisor class virtually in order to simplify some formulas such as the canonical bundle formula of elliptic fibration, the adjunction formula, and the ramification formula. For example, we explain that the arithmetic genus 
$p_{a}(D)=\operatorname{dim} \mathrm{H}^{1}\left(D, \mathcal{O}_{D}\right)$ for a connected reduced divisor $D$ is calculated by $2 p_{a}(D)-2=$ $\left(K_{X}+D\right) \cdot D$, which is derived from the adjunction formula $\left.K_{D} \sim\left(K_{X}+D\right)\right|_{D}$.

\section{Non-ALGEBRAIC SURFACES}

Let $X$ be a non-singular compact complex surface. The algebraic dimension $a(X)$ is the transcendence degree of the meromorphic function field of $X$ over $\mathbb{C}$. Here, $a(X) \leq 1$ if and only if $X$ is non-algebraic. If $a(X)=1$, then the algebraic reduction $\pi: X \rightarrow T$ is holomorphic and is an elliptic fibration. Moreover any curves on $X$ are contained in fibers of $\pi$. If $a(X)=0$, then there exist at most finitely many irreducible curves on $X$ by Theorem 5.1 of $[9, \mathrm{I}]$. We recall the following useful results:

Lemma 2.1. Suppose that $a(X) \leq 1$. Then a line bundle $\mathcal{L}$ of $X$ satisfies the following properties:

(1) $c_{1}(\mathcal{L})^{2} \leq 0$

(2) If $c_{1}(\mathcal{L})^{2}=0$, then $c_{1}(\mathcal{L}) \cdot c_{1}\left(\mathcal{L}^{\prime}\right)=0$ for any line bundle $\mathcal{L}^{\prime}$.

(3) If $p_{g}(X)=0$ and $c_{1}(\mathcal{L})^{2}=0$, then $c_{1}(\mathcal{L})$ is torsion in $\mathrm{H}^{2}(X, \mathbb{Z})$.

Proof. (1) Suppose that $c_{1}(\mathcal{L})^{2}>0$. The Riemann-Roch formula for $\chi\left(X, \mathcal{L}^{\otimes m}\right)$ implies that $\mathrm{h}^{0}\left(X, \mathcal{L}^{\otimes m}\right)$ or $\mathrm{h}^{0}\left(X, \mathcal{L}^{\otimes(-m)} \otimes \omega_{X}\right)$ increases of order $m^{2}$ as $m \rightarrow \infty$. But the former case does not occur since $\kappa(\mathcal{L}, X) \leq a(X) \leq 1$. Thus there exists a non-zero effective divisor $D$ such that $\mathcal{O}_{X}(D) \simeq \omega_{X} \otimes \mathcal{L}^{\otimes(-n)}$ for some $n>0$. The exact sequence

$$
0 \rightarrow \mathrm{H}^{0}\left(X, \mathcal{L}^{\otimes(-m+n)}\right) \rightarrow \mathrm{H}^{0}\left(X, \omega_{X} \otimes \mathcal{L}^{\otimes(-m)}\right) \rightarrow \mathrm{H}^{0}\left(D,\left.\omega_{X} \otimes \mathcal{L}^{\otimes(-m)}\right|_{D}\right)
$$

implies $\kappa\left(\mathcal{L}^{-1}, X\right)=2$ contradicting $\kappa\left(\mathcal{L}^{-1}, X\right) \leq a(X) \leq 1$.

(2) This is shown by (1) and by the inequalities

$$
0 \geq\left(t c_{1}(\mathcal{L})+c_{1}\left(\mathcal{L}^{\prime}\right)\right)^{2}=2 t c_{1}(\mathcal{L}) \cdot c_{1}\left(\mathcal{L}^{\prime}\right)+c_{1}\left(\mathcal{L}^{\prime}\right)^{2}
$$

for any rational number $t$.

(3) follows from (2), from the surjectivity of $c_{1}: \operatorname{Pic}(X) \rightarrow \mathrm{H}^{2}(X, \mathbb{Z})$, and from the non-degeneracy of the intersection pairing on $\mathrm{H}^{2}(X, \mathbb{Q})$.

Notation. Let $C$ be an irreducible curve on a non-singular compact complex surface.

(1) If $C^{2}<0$, then $C$ is called a negative curve.

(2) If $C^{2}=0$, then $C$ is called a 0 -curve.

(3) If $C \simeq \mathbb{P}^{1}$ and $C^{2}=-d<0$, then $C$ is called a $(-d)$-curve.

An exceptional curve of the first kind is just a $(-1)$-curve. If $a(X) \leq 1$, then a nonnegative irreducible curve is a 0 -curve with $p_{a}=1$ and does not intersect other curves.

REmark. A relative minimal model $Y$ of $X$ is, by definition, a non-singular compact complex surface bimeromorphic to $X$ having no $(-1)$-curves. If $X$ is non-algebraic, then $Y$ is unique up to isomorphisms. This is shown as follows: Suppose that there exist a 
bimeromorphic morphism $\mu: X \rightarrow Y$ and a (-1)-curve $C \subset X$ such that $\mu(C)$ is not a point. Then $\mu(C)$ is a 0 -curve with the arithmetic genus $p_{a}(\mu(C))=1$ by Lemma 2.1. Thus $\mu(C)$ has a node or a cusp. Let $Y^{\prime} \rightarrow Y$ be the blowup at the singular point of $\mu(C)$. Then the self-intersection number of the proper transform of $\mu(C)$ is less than -1 . Since $\mu$ factors through $Y^{\prime} \rightarrow Y$, this is a contradiction. Thus, we call $Y$ the minimal model of $X$ in the non-algebraic case. Similarly, a non-algebraic surface without $(-1)$-curves is called a minimal surface.

If $X$ is a non-Kähler elliptic surface with $\kappa(X)=0$, then $b_{1}(X)=3$ or 1 . In the case $b_{1}(X)=3$, the minimal model is the quotient space of $\mathbb{C}^{2}$ by the action of an affine transformation group and is called a primary Kodaira surface. In the case $b_{1}(X)=1$, the minimal model has a primary Kodaira surface as a finite étale covering space and is called a secondary Kodaira surface.

Let $X$ be a compact complex surface with $a(X)=0$. If $b_{1}(X)$ is even, then the minimal model of $X$ is either a complex torus or a K3 surface. If $b_{1}(X)$ is odd, then $b_{1}(X)=1$.

In the classification theory of compact complex surfaces by Kodaira [10], the class VII is not completely classified. A compact complex surface belongs to the class VII if $b_{1}(X)=1$. The class $\mathrm{VII}_{0}$ consists of all the minimal surfaces of class VII. A surface $X$ of class VII has the following invariants:

$$
q(X)-1=p_{g}(X)=\chi\left(X, \mathcal{O}_{X}\right)=h^{1,0}(X)=0, \quad b_{2}(X)=-K_{X}^{2} \geq 0 .
$$

Moreover the intersection pairing on $\mathrm{H}^{2}(X, \mathbb{Q})$ is negative definite.

A Hopf surface is a surface whose universal covering space is isomorphic to $W:=$ $\mathbb{C}^{2} \backslash\{(0,0)\}$, by definition. This is a surface of class $\mathrm{VII}_{0}$ with $b_{2}=0$ containing an elliptic curve.

The classification of surfaces of class $\mathrm{VII}_{0}$ after Kodaira [10] was started by the discovery of Inoue surfaces [5], [6], [7]. The Inoue surfaces $S_{M}, S_{N, p, q, r ; t}^{(+)}, S_{N, p, q, r}^{(-)}$contain no curves and have the vanishing second Betti number. The surfaces $S_{M}$ are also found by Bombieri and are called Bombieri-Inoue surfaces. Inoue [5] showed that if a surface $S$ of class $\mathrm{VII}_{0}$ contains no curves, $b_{2}(S)=0$, and has a line bundle $\mathcal{L}$ with $\mathrm{H}^{0}\left(S, \Omega_{S}^{1} \otimes \mathcal{L}\right) \neq 0$, then $S$ is isomorphic to one of the Inoue surfaces above. The last condition on the existence of $\mathcal{L}$ is not required for the characterization. This was shown by [11], [21] in 1990's. The other Inoue surfaces: Parabolic Inoue surface $X_{\lambda, n}$, Hyperbolic Inoue surface $X_{\mathfrak{K}, \mathrm{N}}$, Half Inoue surface $\widehat{X}_{\mathfrak{K}, \mathrm{N}}$, are constructed in [7]. These surfaces contain curves and have positive second Betti numbers. A parabolic Inoue surface is related to Hirzebruch's cusp singularities and is called also a Hirzebruch-Inoue surface. Another construction of these Inoue surfaces with curves is given in [20] by the method of torus embedding theory (cf. Section 9). 
There are many contributions to the classification of surfaces of class $\mathrm{VII}_{0}$ by Kato [8], Enoki [2], Nakamura [13], [14], and others. The following surfaces are listed in the Table (10.3) of [13]:

FACT. The surfaces $X$ of class $\mathrm{VII}_{0}$ with $a(X)=0$ are classified as follows:

(1) Hopf surface with $a(X)=0$;

(2) A parabolic Inoue surface: It is characterized as a surface containing an elliptic curve and a cycle of rational curves;

(3) A hyperbolic Inoue surface: It is characterized as a surface containing two cycles of rational curves;

(4) An exceptional compactification with no elliptic curves (cf. [2]): It is characterized as a surface containing a cycle $D$ of rational curves with $D^{2}=0$ and no elliptic curves;

(5) A half Inoue surface: It is characterized as a surface containing a cycle $D$ of rational curves with $D^{2}<0$ and $b_{2}(X)=b_{2}(D)$;

(6) A surface with a cycle $D$ of rational curves with $D^{2}<0$ and $b_{2}(X)>b_{2}(D)$;

(7) A surface with no elliptic curves and with no cycles of rational curves.

Here, by a cycle of rational curves, we mean a reduced connected divisor $D=\sum C_{i}$ satisfying one of the following conditions:

(1) $D$ is an irreducible rational curve with exactly one node;

(2) Any irreducible component $C_{i}$ is isomorphic to $\mathbb{P}^{1}$ and intersects with $D-C_{i}$ transversely at two points.

\section{Curves of Negative SELF-INTERSECTiOn NUMBER.}

The argument of this section is almost parallel to that of Section 2 of [19], where the algebraic case was discussed.

Lemma 3.1. A surjective endomorphism $f: X \rightarrow X$ is a finite morphism. If $\kappa(X) \geq 0$, then $f$ is étale.

Proof. If an irreducible curve $C$ is contracted to a point by $f$, then $C^{2}<0$. Since $f_{*}: \mathrm{H}^{2}(X, \mathbb{Q}) \rightarrow \mathrm{H}^{2}(X, \mathbb{Q})$ is isomorphic, no irreducible curve is contracted by $f$. Hence $f$ is finite. Suppose that $\kappa(X) \geq 0$. Then $K_{X} \sim f^{*} K_{X}+R$ for the ramification divisor $R \geq 0$. Thus $K_{X} \sim f^{*} f^{*} K_{X}+f^{*} R+R$. Since $f^{*}: \mathrm{H}^{0}\left(X, m K_{X}\right) \rightarrow \mathrm{H}^{0}\left(X, m K_{X}\right)$ is isomorphic, $R+f^{*} R+\cdots$ is contained in the fixed part of $\left|m K_{X}\right|$. Thus $R=0$.

Lemma 3.2. Let $f: X \rightarrow X$ be a surjective endomorphism. If $C$ is a negative curve, then $f(C)$ is also negative and $f^{-1}(f(C))=C$.

Proof. Assume that $f(C)=f\left(C^{\prime}\right)$ for another irreducible curve $C^{\prime}$. Then $a f_{*} C=a^{\prime} f_{*} C^{\prime}$ for some $a, a^{\prime}>0$. Hence $c_{1}\left(a C-a^{\prime} C^{\prime}\right)=0$ in $\mathrm{H}^{2}(X, \mathbb{Q})$. In particular, $C \cdot C^{\prime}<0$ and thus $C=C^{\prime}$. 
Let $f: X \rightarrow X$ be a non-trivial surjective endomorphism of degree $d>1$. We consider the set $\mathcal{S}(X)$ of all the negative curves on $X$. Then $\mathcal{S}(X)$ is preserved by $f$ and the mapping $\mathcal{S}(X) \ni C \mapsto f(C) \in \mathcal{S}(X)$ is injective. Let $R$ be the ramification divisor of $X$ and let $\mathcal{S}_{0}(X)$ be the set of all the negative curves contained in Supp $R$.

Lemma 3.3. If $C \in \mathcal{S}(X) \backslash \mathcal{S}_{0}(X)$, then $\left|C^{2}\right|>\left|f(C)^{2}\right|$.

Proof. There exist natural numbers $a, b$ such that $f_{*} C=a f(C)$ and $f^{*} f(C)=b C$. Here $b=1$ since $C \not \subset \operatorname{Supp} R$. Thus $a=d$ and $a f(C)^{2}=C^{2}$.

The proof of the following elementary Lemma is left to the reader:

Lemma 3.4. Let $\mathcal{S}$ be a set, $\mathcal{S}_{0}$ a finite subset, and let $h: \mathcal{S} \rightarrow \mathcal{S}$ be an injection. If

$$
\mathcal{S}=\bigcup_{m=1}^{\infty}\left(h^{m}\right)^{-1}\left(\mathcal{S}_{0}\right)
$$

then $\mathcal{S}$ is finite and $h^{k}$ is identical for some $k>0$.

By Lemma 3.3 and Lemma 3.4, we have:

Proposition 3.5. $\mathcal{S}(X)$ is a finite set and there is a natural number $k$ with $f^{k}(C)=C$ for any $C \in \mathcal{S}(X)$.

Hence we assume in what follows that $f(C)=C$ for any $C \in \mathcal{S}(X)$. Then $f^{*} C=a C$ and $f_{*} C=a C$ for a natural number $a>1$ with $a^{2}=d$. Let $N_{X}$ denote the reduced divisor $\sum_{C \in \mathcal{S}(X)} C$. Then $R=(a-1) N_{X}+\Delta$ for an effective divisor $\Delta$ whose irreducible component are not negative curves. In particular

$$
K_{X}+N_{X}=f^{*}\left(K_{X}+N_{X}\right)+\Delta .
$$

For any connected reduced curve $D \leq N_{X}$, we have

$$
K_{D}+\left.\left(N_{X}-D\right)\right|_{D}=\left(\left.f\right|_{D}\right)^{*}\left(K_{D}+\left.\left(N_{X}-D\right)\right|_{D}\right)+\left.\Delta\right|_{D}
$$

In particular, $p_{a}(D)=\mathrm{h}^{1}\left(D, \mathcal{O}_{D}\right) \leq 1$. If $p_{a}(D)=1$, then $\Delta \cap D=\left(N_{X}-D\right) \cap D=\emptyset$. If $p_{a}(D)=0$, then $\left(N_{X}-D\right) \cdot D \leq 2$, and if further $\left(N_{X}-D\right) \cdot D=2$, then $\Delta \cap D=\emptyset$.

The induced morphism $\left.f\right|_{D}: D \rightarrow D$ is an endomorphism of degree $a$. Moreover it is étale outside Sing $\left.D \cup \Delta\right|_{D}$ by the well-known Lemma 3.6 below. In particular, $f(\operatorname{Sing} D) \subset$ Sing $\left.D \cup \Delta\right|_{D}$, and $\left.\Delta\right|_{D}$ gives the ramification divisor of $\left.f\right|_{D}$ over $D \backslash \operatorname{Sing} D$.

LemMA 3.6. Let $\tau: U \rightarrow V$ be a finite morphism between non-singular complex manifolds and let $C \subset V$ be a non-singular divisor such that $\tau$ is étale outside $\tau^{-1} C$. Then $\tau^{-1} C \rightarrow C$ is étale.

Proof. We may assume that $V$ is a $d$-dimensional polydisc and $C$ is a hyperplane by considering the local situation. Then $V \backslash C$ is isomorphic to the product of the punctured disc and a $(d-1)$-dimensional polydisc. In particular, the finite étale covering $U \backslash \tau^{-1} C \rightarrow$ $V \backslash C$ is cyclic and $U \rightarrow V$ is the cyclic covering branched along $C$. Hence $\tau^{-1} C \simeq C$. 
A reduced connected divisor $D$ is called a straight chain of rational curves if $D=\sum_{i=1}^{l} C_{i}$ for irreducible curves $C_{i} \simeq \mathbb{P}^{1}$ such that

(1) $C_{i} \cdot C_{j}=0$ for $|i-j| \geq 2$,

(2) $C_{i} \cap C_{j}=1$ for $|i-j|=1$.

Lemma 3.7. A negative curve $C$ is either an elliptic curve, a rational curve with exactly one node, or a smooth rational curve. A reducible connected component of $N_{X}$ is an elliptic curve, a straight chain of rational curves, or a cycle of rational curves.

Proof. If $p_{a}(D)=1$ for a connected reduced curve $D \leq N_{X}$, then $K_{D}=\left(\left.f\right|_{D}\right)^{*} K_{D}$ and $\left.f\right|_{D}: D \rightarrow D$ is étale outside Sing $D$. Thus no rational curves with cusps are negative. If a negative curve $C_{1}$ intersects another negative $C_{2}$ at one point not transversely, then $p_{a}\left(C_{1}+C_{2}\right)=1$. This contradicts the property: no étale covering exists over $C_{1} \backslash C_{2} \simeq \mathbb{C}$. If three negative curves $C_{1}, C_{2}, C_{3}$ intersect transversely as $C_{1} \cap C_{2}=C_{2} \cap C_{3}=C_{3} \cap C_{1}=$ $\{P\}$ for a point $P$, then $p_{a}\left(C_{1}+C_{2}+C_{3}\right)=1$. This contradicts the same property as above. These observations tell us that a reducible connected component $D$ is a straight chain of rational curves or a cycle of rational curves.

Suppose that $X$ contains a $(-1)$-curve $C$. Let $X \rightarrow X_{1}$ be the blowing down of $C$. Then an endomorphism of $X_{1}$ is induced since $f^{-1} C=C$. Therefore, an endomorphism is induced on a relative minimal model of $X$.

\section{The CASE OF ELLIPTIC SURFACES}

Let $X$ be a non-singular compact complex surface admitting a non-trivial surjective endomorphism. Assume that $a(X)=1$. Let $\pi: X \rightarrow T$ be the algebraic reduction which is an elliptic fibration onto a non-singular projective curve. A non-trivial surjective endomorphism $f$ induces a surjective endomorphism $h$ of $T$ such that $h \circ \pi=\pi \circ f$.

Proposition 4.1. Under the situation, $X$ is a minimal elliptic surface with $e(X)=0$.

Proof. The set of all the irreducible component of reducible fibers coincides with $\mathcal{S}(X)$. A 0 -curve is the support of an irreducible fiber. We may assume that $f^{-1} C=C$ for negative curves $C$ for the endomorphism $f$.

Step 1. We may assume that $f^{-1} C=C$ for any rational curves $C$.

We have to consider only rational 0-curves $C$. If $C^{\prime}$ is an irreducible component of $f^{-1} C$, then $C^{\prime}$ is not negative and $C^{\prime} \rightarrow C$ is étale outside Sing $C$ by Lemma 3.6. If $C$ is a rational curve with a cusp, then $C^{\prime} \simeq C$. If $C$ is a rational curve with a node, then $C^{\prime}$ also has a node since $f$ is branched along the normal crossing divisor around the node. The number of rational 0 -curves are finite. Hence $f^{-1} C$ is irreducible and $\left(f^{k}\right)^{-1} C=C$ for some $k>0$.

Step 2. X admits no curves with cusps. 
Suppose that there exist an irreducible curve $C$ with a cusp and set $P=\pi(C)$. Note that $C=\pi^{*} P$ is a singular fiber of type II. By the argument of Step 1, we infer that $f^{*} C=d C$ for $d=\operatorname{deg} f$. Hence $h^{*} P=d P$. In particular, $\operatorname{deg} h=d$. If $D$ is a connected component of $N_{X}$, then $D=\pi^{-1} P^{\prime \prime}$ and $h^{*} P^{\prime \prime}=d P^{\prime \prime}=a P^{\prime \prime}$ for $a^{2}=d$. Thus $N_{X}=0$. In particular, $\pi$ is a minimal elliptic fibration with only irreducible fibers. If $C^{\prime}=\pi^{-1}\left(P^{\prime}\right)$ is another rational 0-curve, then $h^{*} P^{\prime}=d P^{\prime}$ since $f^{-1} C^{\prime}=C^{\prime}$. Considering the ramification formula for $h$, we infer that $T \simeq \mathbb{P}^{1}$ and there exist at most two rational curves on $X$. If $C$ is the unique rational curve, then $\pi$ is smooth outside $P$ and the local constant system $\left.R^{1} \pi_{*} \mathbb{Z}_{X}\right|_{C \backslash P}$ is trivial. The local monodromy corresponding to a singular fiber of type II is of order 6 in $\mathrm{SL}(2, \mathbb{Z})$. This is a contradiction. Hence there is another rational 0-curve $C^{\prime}=\pi^{-1}\left(P^{\prime}\right)$. If $C^{\prime}$ has a node, then $J\left(P^{\prime}\right)=\infty$ for the $J$-function associated with $\pi$. However, $\pi$ is smooth over $T \backslash\left\{P, P^{\prime}\right\} \simeq \mathbb{C} \backslash\{0\}$. Thus the period function is constant, a contradiction. Hence there remains the case in which $C^{\prime}$ has a cusp. Let $\mathcal{U}$ and $\mathcal{U}^{\prime}$ respectively be open discs with centers $P$ and $P^{\prime}$. A positive generator of $\pi_{1}(\mathcal{U} \backslash\{P\}) \simeq \mathbb{Z}$ corresponds to a negative generator of $\pi_{1}\left(\mathcal{U}^{\prime} \backslash\left\{P^{\prime}\right\}\right)$ by the isomorphisms

$$
\pi_{1}(\mathcal{U} \backslash\{P\}) \rightarrow \pi_{1}\left(T \backslash\left\{P, P^{\prime}\right\}\right) \leftarrow \pi_{1}\left(\mathcal{U}^{\prime} \backslash\left\{P^{\prime}\right\}\right) .
$$

Thus the condition that $C$ is of type II implies that $C^{\prime}$ is of type $\mathrm{II}^{*}$, a contradiction.

Step 3. $X$ admits no rational curves

Assume the contrary. By Step $1, f^{*} C=(\operatorname{deg} h) C$ for any rational curve $C$ on $X$. If $\operatorname{deg} h=1$, then $N_{X}=0$ and $f$ is étale along $f^{-1} C$ for a rational 0 -curve $C$. Here, the mapping degree of $f^{-1} C \rightarrow C$ is $\operatorname{deg} f$. However, there exists only one point in $f^{-1} C$ over the node of $C$. This is a contradiction. Consequently, $\operatorname{deg} h \geq 2$. By the same argument as Step 2, we infer that $T \simeq \mathbb{P}^{1}$ and that the number of singular fibers supported on a union of rational curves is at most 2. Then the period map of $\pi$ is constant. Hence no singular fibers of type ${ }_{m} \mathrm{I}_{b}$ with $b>0$ appear on the relative minimal model of $\pi: X \rightarrow T$. Therefore, $X$ has no rational curves.

As a result, $\pi$ is minimal and a singular fiber is a multiple of an elliptic curve.

The elliptic fibration $\pi: X \rightarrow T$ above defines a variation of Hodge structure $H$ of weight 1 on $T$ since the local monodromies around the image of singular fibers are trivial. Here, we have $R^{1} \pi_{*} \mathbb{Q}_{X} \simeq H \otimes \mathbb{Q}$ (cf. Lemma 5.4.4 of [18]). Here, $\mathrm{H}^{0}(T, H) \neq 0$ implies $H \simeq \mathbb{Z}_{T}^{\oplus 2}$ by Corollary 4.2.5 of [18] (cf. Theorem 11.7 of [9, III]). From Leray's exact sequence

$$
0 \rightarrow \mathrm{H}^{1}(T, \mathbb{Q}) \rightarrow \mathrm{H}^{1}(X, \mathbb{Q}) \rightarrow \mathrm{H}^{0}(T, H \otimes \mathbb{Q}) \rightarrow \mathrm{H}^{2}(T, \mathbb{Q}) \rightarrow \mathrm{H}^{2}(X, \mathbb{Q}),
$$

we infer that $b_{1}(X)$ is odd if and only if $H$ is trivial and $\mathrm{H}^{2}(T, \mathbb{Q}) \rightarrow \mathrm{H}^{2}(X, \mathbb{Q})$ is zero. If $b_{1}(X)$ is even, then $X$ is Kähler by Miyaoka [12]. Let $\mathcal{L}$ be the invertible sheaf $R^{1} \pi_{*} \mathcal{O}_{X}$. Then $\mathcal{L}$ is isomorphic to the graded piece $\mathrm{Gr}^{0}$ for the Hodge filtration on $H \otimes \mathcal{O}_{T}$ and 
$\pi_{*} \omega_{X} \simeq \omega_{T} \otimes \mathcal{L}^{-1}$. Moreover, $\mathcal{L}^{\otimes 12} \simeq \mathcal{O}_{X}$. Then $g(T) \leq q(X)=g(T)+\mathrm{h}^{0}(T, \mathcal{L}) \leq g(T)+1$ by

$$
0 \rightarrow \mathrm{H}^{1}\left(T, \mathcal{O}_{T}\right) \rightarrow \mathrm{H}^{1}\left(X, \mathcal{O}_{X}\right) \rightarrow \mathrm{H}^{0}(T, \mathcal{L}) \rightarrow 0 .
$$

Hence $p_{g}(X)=g(T)-1+\mathrm{h}^{0}(T, \mathcal{L})$ by $\chi\left(X, \mathcal{O}_{X}\right)=0$. If $\mathrm{h}^{0}(T, \mathcal{L})=0$, then $\mathrm{h}^{0}(T, H)=0$, $b_{1}(X)=2 g(T)$, and $X$ is Kähler. If $h^{0}(T, \mathcal{L}) \neq 0$, or equivalently, $\mathcal{L} \simeq \mathcal{O}_{T}$, then the Weierstrass model [15] associated with $H$ is isomorphic to the product of an elliptic curve and $T$, and hence $H$ is trivial.

Lemma 4.2. The induced endomorphism $h: T \rightarrow T$ is not identical.

Proof. Assume the contrary. Then $f$ is an endomorphism over $T$. Let $\Sigma$ be a set of points $P \in T$ such that $\pi^{*} P$ is a multiple fiber. Let $m_{P}$ be the multiplicity of $\pi^{*} P$. Then we have a finite ramified covering $\tau: Z \rightarrow T$ such that $\tau^{*} P=m_{P}\left(\tau^{*} P\right)_{\text {red }}$ for $P \in \Sigma$ and $g(Z) \geq 2$. Then the normalization of $X \times_{T} Z$ is smooth over $Z$ and admits a non-trivial surjective endomorphism. Thus we may assume from the first that $\pi$ is smooth and $g(T) \geq 2$. By considering the étale cyclic covering given by $\mathcal{L}^{\otimes k} \simeq \mathcal{O}_{T}$, we may also assume that $\mathcal{L} \simeq \mathcal{O}_{T}$ and hence the variation of Hodge structure $H$ is trivial. Let $E$ be the elliptic curve isomorphic to a fiber of $\pi$. We fix a point $0 \in E$ and give a group structure on $E$ whose zero is 0 . Let $\mathcal{O}_{T}(E)$ be the sheaf of germs of holomorphic mappings from $T$ to $E$. Then we have an exact sequence

$$
0 \rightarrow H \simeq \mathbb{Z}_{T}^{\oplus 2} \rightarrow \mathcal{O}_{T} \rightarrow \mathcal{O}_{T}(E) \rightarrow 0
$$

There is an element $\eta \in H^{1}\left(T, \mathcal{O}_{T}(E)\right)$ such that $\pi$ is obtained as the torsor of $E \times T$ over $T$ defined by $\eta$. The endomorphism $f$ induces an endomorphism $f_{*}: H \rightarrow H$ of variation of Hodge structures which corresponds to

$$
H^{1}\left(\pi^{-1}(P), \mathbb{Z}\right) \simeq H_{1}\left(\pi^{-1}(P), \mathbb{Z}\right) \stackrel{f_{*}}{\rightarrow} H_{1}\left(\pi^{-1}(P), \mathbb{Z}\right) \simeq H^{1}\left(\pi^{-1}(P), \mathbb{Z}\right),
$$

where the edge isomorphisms are the Poincaré duals. The endomorphism $f_{*}: E \rightarrow E$ keeps 0 and is the multiplication by a complex number $\lambda$. If we identify $E$ as the quotient of $\mathbb{C}$ by the lattice $L_{\theta}=\mathbb{Z} \theta+\mathbb{Z}$ for some $\theta \in \mathbb{H}$, then $\lambda L_{\theta} \subset L_{\theta}$. Hence $1 \neq \lambda \in \mathbb{Z}$ or $\mathbb{Q}(\lambda)$ is an imaginary quadratic field. In the latter case, $t=\lambda+\bar{\lambda}$ and $d=|\lambda|^{2}$ are integers with $1-t+d \neq 0$. The cohomology class $\eta$ satisfies $\lambda_{*} \eta=\eta$. Hence $(\lambda-1) \eta=0$ or $(1-t+d) \eta=0$. Thus $\eta$ is torsion, which implies that $\pi$ is projective. This contradicts $a(X)=1$.

Corollary 4.3. $g(T) \leq 1$. If $g(T)=1$, then $\pi$ is smooth.

Proof. If $g(T) \geq 2$, then $h^{k}=\mathrm{id}_{T}$ for some $k>0$. If $g(T)=1$ and if there exists a multiple fiber $F=\pi^{-1}(P)$, then $\pi^{-1}(Q)$ is also multiple for any $Q \in h^{-1}(P)$, since $h: T \rightarrow T$ is étale. Thus $h$ is isomorphic and $h^{k}$ keeps $P$ for some $k>0$ since the set of multiple fibers is finite. Hence $h^{k l}$ is identical for some $l>0$, since the group of automorphisms of $E$ keeping $P$ is finite. 
Lemma 4.4. Let $\pi: X \rightarrow T$ be an elliptic surface of class $\mathrm{VII}_{0}$. Then $T \simeq \mathbb{P}^{1}$ and singular fibers are multiple of elliptic curves. In particular, $K_{X} \sim_{\mathbb{Q}} \pi^{*}\left(K_{T}+\Theta\right)$ for an effective $\mathbb{Q}$-divisor $\Theta=\sum\left(1-m_{i}^{-1}\right) P_{i}$ on $T$, where $m_{i}$ is the multiplicity of the fiber $\pi^{*} P_{i}$. Furthermore the following assertions hold:

(1) If $\operatorname{deg} \Theta>2$, then any surjective endomorphism of $X$ is isomorphic.

(2) If $\operatorname{deg} \Theta=2$, then $X$ is a secondary Kodaira surface.

(3) If $\operatorname{deg} \Theta<2$, then $X$ is an elliptic Hopf surface.

Proof. $T$ is rational by $b_{1}(T) \leq b_{1}(X)=1$. The variation of Hodge structure is trivial since $e(X)=0$ and $\pi_{1}(T)=\{1\}$. Let $\Sigma=\left\{P_{1}, P_{2}, \ldots\right\}$ be the set of points $P$ such that $\pi^{*} P$ is a multiple fiber. Then $\pi^{*} P_{i}=m_{i} C_{i}$ for an elliptic curve $C_{i}$ and $m_{i} \geq 2$. We assume that $m_{1} \leq m_{2} \leq \cdots$ Then

$$
K_{X} \sim \pi^{*} K_{T}+\sum\left(m_{i}-1\right) C_{i} \sim_{\mathbb{Q}} \pi^{*}\left(K_{T}+\Theta\right), \quad \text { for } \quad \Theta=\sum\left(1-\frac{1}{m_{i}}\right) P_{i} .
$$

In particular, $\kappa(X)=1,0,-\infty$ according as $\operatorname{deg} \Theta>2,=2,<2$.

Suppose that $\kappa(X)=1$. Let $f$ be a surjective endomorphism of $X$ and $h$ the induced endomorphism of $T$ with $\pi \circ f=h \circ \pi$. Then $f$ is étale by Lemma 3.1. Thus $K_{X} \sim f^{*} K_{X}$ implies that $K_{T}+\Theta \sim_{\mathbb{Q}} h^{*}\left(K_{T}+\Theta\right)$. Thus $h$ is an automorphism keeping the set $\Sigma$ which consists at least three points. Hence some power $h^{k}$ is identical and $f$ is isomorphic by Lemma 4.2.

Suppose that $\kappa(X)=0$. Then $\left(m_{1}, m_{2}, \ldots\right)$ is one of the followings:

$$
(2,2,2,2), \quad(2,3,6), \quad(2,4,4), \quad(3,3,3) .
$$

In each case, there is a cyclic covering $\tau: A \rightarrow T$ from an elliptic curve such that $\tau^{*} P_{i}=$ $m_{i}\left(\tau^{*} P_{i}\right)_{\text {red }}$ for any $i$ and that $\tau$ is étale outside $\Sigma$. Moreover, for a suitable choice of group structure of $A$, a generator of the Galois group of $\tau$ is given as the multiplication map $z \mapsto \alpha z$ by a primitive root $\alpha$ of unity of order $2,6,4,3$ according as $(2,2,2,2),(2,3,6)$, $(2,4,4),(3,3,3)$ above. The normalization $Y$ of the fiber product $X \times_{T} A$ is smooth over $A$ and étale over $X$. Hence $Y$ is a primary Kodaira surface and $X$ is secondary.

Finally suppose that $\operatorname{deg} \Theta<2$. If $\Sigma \neq \emptyset$, then $\left(m_{1}, m_{2}, \ldots\right)$ is one of the followings:

$$
\left(m_{1}\right), \quad\left(m_{1}, m_{2}\right), \quad\left(2,2, m_{3}\right), \quad(2,3,3), \quad(2,3,4), \quad(2,3,5) .
$$

If $\sharp \Sigma \leq 2$, then $X$ is Hopf by Lemma 8 of [10] (cf. Fact 7.2 below). If $\sharp \Sigma=3$, then there is a finite Galois covering $\tau: \Gamma \rightarrow T$ from a non-singular rational curve $\Gamma$ such that $\tau^{*} P_{i}=m_{i}\left(\tau^{*} P_{i}\right)_{\text {red }}$ for any $i$ and that $\tau$ is étale outside $\Sigma$. Moreover, $\tau$ is isomorphic to the quotient morphism by the standard action of the following finite group $G \subset \operatorname{Aut}(\Gamma)$ according to $(2,2, n),(2,3,3),(2,3,4),(2,3,5)$ : the dihedral group $D_{n}$ of order $2 n$, the tetrahedral group $\mathfrak{A}_{4}$, the octahedral group $\mathfrak{S}_{4}$, and the icosahedral group $\mathfrak{A}_{5}$. The normalization $Y$ of the fiber product $X \times_{T} \Gamma$ is smooth over $\Gamma$ and étale over $X$. Hence $X$ is also a Hopf surface since $Y$ is so. 
Theorem 4.5. Let $X$ be a non-singular compact complex surface admitting a nontrivial surjective endomorphisms. If $a(X)=1$, then $X$ is a complex torus, a primary Kodaira surface, a secondary Kodaira surface or an elliptic Hopf surface.

Proof. Assume that $g(T)=1$. If $H$ is not trivial, then $X$ is Kähler and $p_{g}(X)=0$. This implies that $X$ is projective, a contradiction. Hence $H$ is trivial. Thus $\omega_{X} \simeq \mathcal{O}_{X}$ and $3 \leq b_{1}(X) \leq 4$. If $b_{1}(X)=4$, then $X$ is a complex torus. If $b_{1}(X)=3$, then $X$ is a primary Kodaira surface.

Next assume that $g(T)=0$. Then $\mathcal{L} \simeq \mathcal{O}_{T}$ and $H$ is trivial. In particular, $p_{g}(X)=0$ and $q(X)=1$. Thus $X$ is a surface of class $\mathrm{VII}_{0}$. It is a Hopf surface or a secondary Kodaira surface by Lemma 4.4 .

\section{Appendix to SECTION 4}

The existence of non-trivial surjective endomorphisms on an algebraic surface $X$ with $\kappa(X)=1, e(X)=0$ is proved in Proposition 3.3 of [3] by using the $\partial$-étale cohomology theory developed in [18]. Here, we shall give a more geometric proof.

Let $\pi: X \rightarrow T$ be the elliptic fibration obtained as the Iitaka fibration. Let $\Sigma$ be the set of points $P \in T$ such that $\pi^{*} P$ is a multiple fiber of multiplicity $m_{P} \geq 2$. Then $K_{X} \sim_{\mathbb{Q}} \pi^{*}\left(K_{T}+\Theta\right)$ for the $\mathbb{Q}$-divisor $\Theta=\sum_{P \in \Sigma}\left(1-m_{P}^{-1}\right) P$ as in Lemma 4.4. Note that $\operatorname{deg}\left(K_{T}+\Theta\right)>0$ by $\kappa(X)=1$. By applying Theorem 4.2 of [16], we have a finite Galois covering $Z \rightarrow T$ such that the normalization $Y$ of $X \times_{T} Z$ is isomorphic to the product $C \times Z$ over $Z$ for an elliptic curve $C$ and is étale over $X$. We consider $C$ as the torus $\mathbb{C} / L$ for the lattice $L=\mathbb{Z} \tau+\mathbb{Z}$ with $\operatorname{Im} \tau>0$. We denote by $[x]$ the image of $x \in \mathbb{C}$ under $\mathbb{C} \rightarrow C$. Let $G$ be the Galois group. Then the induced action of $g \in G$ on $Y \simeq C \times Z$ is written by

$$
([x], z) \mapsto\left(\left[a_{g} x\right]+b_{g}(z), g \cdot z\right)
$$

for some $a_{g} \in \mathbb{C}^{\star}$ and some holomorphic mapping $b_{g}: Z \rightarrow C$. Here, $\left\{a_{g}\right\}$ gives rise to a homomorphism $G \rightarrow \mathbb{C}^{\star}$ and $L$ is a $G$-submodule of $\mathbb{C}$. In particular, the complex torus $C$ is a $G$-module. The set $\operatorname{Hom}(Z, C)$ of holomorphic maps $\varphi: Z \rightarrow C$ also has a right $G$-module structure by $\varphi^{g}(z)=a_{g}^{-1} \varphi(g \cdot z)$. By the relation $a_{g} b_{h}(z)+b_{g}(h \cdot z)=b_{g h}(z)$ for $g, h \in G$, we infer that $\left\{a_{g}^{-1} b_{g}\right\}$ defines an element of $H^{1}(G, \operatorname{Hom}(Z, C))$. Since the cohomology group is torsion, there exist a positive integer $n$ and a holomorphic mapping $c: Z \rightarrow C$ such that

$$
n a_{g}^{-1} b_{g}(z)=c(z)-a_{g}^{-1} c(g \cdot z)
$$

for any $g \in G$. The endomorphism $C \times Z \rightarrow C \times Z$ given by

$$
([x], z) \mapsto((n+1)[x]+c(z), z)
$$

commutes with the action of $G$ on $C \times Z$. Thus it induces a non-trivial surjective endomorphism on $X$. 


\section{The CASe of Algebraic dimension zero}

Let $X$ be a non-singular compact complex surface of $a(X)=0$ admitting a nontrivial surjective endomorphism. Suppose that $X$ is Kähler. Then $\kappa(X)=0$. Thus the endomorphism is étale and hence $X$ admits no negative curves. Hence $X$ is minimal and is a complex torus. A complex torus admits a non-trivial surjective endomorphism as the multiplication map by an integer greater than 1 .

Thus we assume that $X$ is non-Kähler. Then $X$ belongs to the class VII. We have $\left(K_{X}+N_{X}\right)^{2}=0$ by (3.1). Thus $p_{a}(D)=1$ for any connected component $D$ of $N_{X}$. Moreover, $K_{X}^{2}=N_{X}^{2}=\sum D_{\lambda}^{2}$ for the decomposition $N_{X}=\sum D_{\lambda}$ into the connected components.

Lemma 5.1. If $D$ is a reduced divisor with $\left(K_{X}+D\right) \cdot D=0$, then $D$ has at most two connected components.

Proof. Since $a(X)=0$, we have $\mathrm{h}^{0}\left(X, \mathcal{O}_{X}\left(K_{X}+D\right)\right)=\mathrm{h}^{2}\left(X, \mathcal{O}_{X}(-D)\right) \leq 1$. Hence $\mathrm{h}^{1}\left(X, \mathcal{O}_{X}(-D)\right) \leq 1$ by $\left(K_{X}+D\right) \cdot D=0$. The exact sequence

$$
0 \rightarrow \mathrm{H}^{0}\left(X, \mathcal{O}_{X}\right) \rightarrow \mathrm{H}^{0}\left(X, \mathcal{O}_{D}\right) \rightarrow \mathrm{H}^{1}\left(X, \mathcal{O}_{X}(-D)\right)
$$

implies $\mathrm{h}^{0}\left(D, \mathcal{O}_{D}\right) \leq 2$.

TheOrem 5.2. Let $X$ be a non-Kähler surface of $a(X)=0$ admitting a non-trivial surjective endomorphism. Then the minimal model of $X$ is one of the following surfaces: a parabolic Inoue surface; a hyperbolic Inoue surface; a half Inoue surface; a Hopf surface; an Inoue surface with no curves. Moreover, $X$ is obtained from the minimal model by a succession of blowups whose centers are nodes of curves.

Proof. One of the following cases occurs by Lemma 5.1:

Case 1. $N_{X}$ has two connected components;

Case 2. $N_{X}$ is connected;

Case 3. $X$ contains a 0 -curve but no negative curves;

Case 4. $X$ contains no curves.

Let $Y$ be the minimal model of $X$ and let $\mu: X \rightarrow Y$ be the contraction. Then the endomorphism of $X$ descends to $Y$ and $N_{Y} \leq \mu_{*} N_{X}$.

Case 1. Any curve on $X$ is contained in $N_{X}$ by Lemma 5.1. Thus $f^{*}\left(K_{X}+N_{X}\right) \sim$ $K_{X}+N_{X}$. We have $\mathrm{h}^{2}\left(X, \mathcal{O}_{X}\left(-N_{X}\right)\right)=1$ by the exact sequence

$$
\mathrm{H}^{1}\left(X, \mathcal{O}_{X}\right) \rightarrow \mathrm{H}^{1}\left(N_{X}, \mathcal{O}_{N_{X}}\right) \rightarrow \mathrm{H}^{2}\left(X, \mathcal{O}_{X}\left(-N_{X}\right)\right) \rightarrow \mathrm{H}^{2}\left(X, \mathcal{O}_{X}\right)=0 .
$$

Thus $K_{X}+N_{X} \sim E$ for an effective divisor $E$. Here $f^{*} E=E$. Therefore, $E=0$, equivalently, $K_{X}+N_{X} \sim 0$. Let $D_{1}$ and $D_{2}$ be the two connected components of $\mu_{*} N_{X} \sim$ $-K_{Y}$. Then $p_{a}\left(D_{i}\right)=1$ for $i=1,2$. By Lemma (2.11) of [13], $D_{1}$ is an elliptic curve if and only if $D_{2}^{2}=0$. Hence, if $D_{2}^{2}=0$, then $D_{1}^{2}<0$. Otherwise, $D_{1}$ and $D_{2}$ are both 
elliptic curves and $\mu_{*} N_{X}$ has no nodes, which implies that $\mu$ is isomorphic and $N_{X}=0$, a contradiction. Therefore if $D_{1}^{2}=0$ or $D_{2}^{2}=0$, then $Y$ is a parabolic Inoue surface by Theorem (7.1) of [13] (cf. (7.12) of [13], [2]). If $D_{1}^{2}<0$ and $D_{2}^{2}<0$, then $N_{Y}=\mu_{*} N_{X}$ and $Y$ is a parabolic Inoue surface by Theorem (8.1) of [13]. In both cases, $\mu: X \rightarrow Y$ is a successive blowups whose centers are nodes.

Case 2. Suppose that there is a curve $C$ not contained in $N_{X}$. Then any curve on $X$ is contained in $C \cup N_{X}$. The contraction $\mu: X \rightarrow Y$ is isomorphic along $C$. Since $C^{2}=0$, then $\mu_{*} N_{X}$ is an elliptic curve and $C$ is a rational curve with a node by Lemma (2.11) of [13]. Then $\mu$ is isomorphic and $X$ is a parabolic Inoue surface of $b_{2}=1$ by [2] or by Theorem (7.1) of [13].

Next suppose that any curve on $X$ is contained in $N_{X}$. Then $f^{*}\left(K_{X}+N_{X}\right) \sim K_{X}+$ $N_{X}$. Moreover, $f$ induces a finite étale endomorphism on the complement $U=X \backslash N_{X}$. Therefore, $e(U)=0$. Thus $e(X)=e\left(N_{X}\right)$. If $N_{X}$ is an elliptic curve, then $-N_{X}^{2}=-K_{X}^{2}=$ $e(X)=0$, a contradiction. Thus $N_{X}$ is a cycle of rational curves. Here, $e(X)=e\left(N_{X}\right)$ is equivalent to $b_{2}(X)=b_{2}\left(N_{X}\right)$. Thus $b_{2}(Y)=b_{2}\left(\mu_{*} N_{X}\right)$. If $N_{Y} \neq 0$, then $N_{Y}=\mu_{*} N_{X}$ and $Y$ is a half Inoue surface by [13]. If $N_{Y}=0$, then $\mu_{*} N_{X}$ is a rational curve with a node. This case does not occur by the argument in Case 3 below. Therefore, $X$ is obtained as a successive blowups of a half Inoue surface whose centers are nodes.

Case 3. We have $b_{2}(X)=e(X)=-K_{X}^{2}=-\left(K_{X}+N_{X}\right)^{2}=0$. By Lemma (2.11) of [13], one of the following three possibilities remain:

(1) $X$ contains two elliptic curves;

(2) $X$ contains an elliptic curve as a unique curve.

(3) $X$ contains a rational curve with a node as a unique curve.

For the complement $U$ of the union of all the curves on $X$, we have $e(U)=0$ since $f$ induces a finite étale endomorphism on $U$. Hence the case (3) does not occur by $e(X \backslash U)=0$. In the cases (1), (2), $X$ is a Hopf surface by Lemma 8 of [10].

Case 4. Since $b_{2}(X)=-K_{X}^{2}=0, X$ is one of Inoue surfaces without curves by [5], $[11],[21]$.

\section{KodAira SURFACES}

A primary Kodaira surface $X$ is defined as a surface with $K_{X} \sim 0, b_{1}(X)=3$. The algebraic reduction $\pi: X \rightarrow T$ is an elliptic fibration over an elliptic curve $T$. This is smooth by $e(X)=0$ and $K_{X} \sim 0$. Moreover the associated variation of Hodge structure $H$ is trivial since $\pi_{*} \omega_{X / T} \simeq \mathcal{L}^{-1} \simeq \mathcal{O}_{T}$. For a fiber $E$, we fix a point 0 and give an abelian group structure on $E$ with 0 being the identity. Then as in the proof of Lemma 4.2, $X \simeq(E \times T)^{\eta}$ as a torsor corresponding to some $\eta \in \mathrm{H}^{1}\left(T, \mathcal{O}_{T}(E)\right)$, where $\mathcal{O}_{T}(E)$ is the sheaf of germs of holomorphic mappings from $T$ to $E$. The image of $\eta$ under $\mathrm{H}^{1}\left(T, \mathcal{O}_{T}(E)\right) \rightarrow \mathrm{H}^{2}(T, H)=\mathrm{H}^{2}\left(T, \mathbb{Z}^{2}\right)$ is not zero, since $X$ is non-Kähler. 
Let $L_{\tau}$ denote the lattice $\mathbb{Z} \tau+\mathbb{Z} \subset \mathbb{C}$ for $\tau \in \mathbb{H}$. We fix $\tau, \theta \in \mathbb{H}$ and isomorphisms $T \simeq \mathbb{C} / L_{\tau}, E \simeq \mathbb{C} / L_{\theta}$. For $c \in L_{\theta}$ and $\delta \in \mathbb{C}$, let us consider the following automorphisms of $\mathbb{C} \times E$ :

$$
g_{1}:(z,[\zeta]) \mapsto(z+\tau,[\zeta+c z+\delta]), \quad \text { and } \quad g_{2}:(z,[\zeta]) \mapsto(z+1,[\zeta]),
$$

where $[\zeta]$ denotes $\zeta \bmod L_{\theta}$. The quotient space of $\mathbb{C} \times E$ by $g_{1}$ and $g_{2}$ is denoted by $X_{c, \delta}$. Let $\pi: X_{c, \delta} \rightarrow T$ denote the induced smooth elliptic fibration from the first projection $\mathbb{C} \times E \rightarrow \mathbb{C}$.

Lemma 6.1. A primary Kodaira surface is isomorphic to $X_{c, \delta}$ for some $c \neq 0$ and $\delta$.

Proof. We have an isomorphism $\mathrm{H}^{1}\left(T, \mathcal{O}_{T}(E)\right) \simeq \mathrm{H}^{1}\left(L_{\tau}, \mathrm{H}^{0}(\mathbb{C}, \mathcal{O}(E))\right)$ by HochschildSerre spectral sequence for the universal covering map $\mathbb{C} \rightarrow T$. Thus the cohomology class $\eta$ is represented by a cocycle $\left\{x_{u}=x_{u}(z)\right\}$ of holomorphic functions on $\mathbb{C}$ for $u \in L_{\tau}$ such that $x_{u+v}(z) \equiv x_{v}(z)+x_{u}(z+v) \bmod L_{\theta}$. Here, $X$ is isomorphic to the quotient space of $\mathbb{C} \times E$ by the following action of $u \in L_{\tau}$ :

$$
(z,[\zeta]) \mapsto\left(z+u,\left[\zeta+x_{u}(z)\right]\right) .
$$

Thus we shall find a simple form of $x_{u}(z)$ up to coboundary. Note that $\left\{x_{u}\right\}$ is determined only by $x_{1}$ and $x_{\tau}$ which satisfy

$$
x_{\tau}(z+1)-x_{\tau}(z) \equiv x_{1}(z+\tau)-x_{1}(z) \bmod L_{\theta}
$$

We know that $\operatorname{dim} \mathrm{H}^{1}\left(T, \mathcal{O}_{T}\right)=1$ and $\mathrm{H}^{1}(T, \mathbb{C}) \rightarrow \mathrm{H}^{1}\left(T, \mathcal{O}_{T}\right)$ is surjective. The homomorphism is isomorphic to $\mathrm{H}^{1}\left(L_{\tau}, \mathbb{C}\right) \rightarrow \mathrm{H}^{1}\left(L_{\tau}, \mathrm{H}^{0}(\mathbb{C}, \mathcal{O})\right)$. Hence, for a cocycle $\left\{y_{u}(z)\right\}$ of holomorphic functions on $\mathbb{C}$ satisfying $y_{u+v}(z)=y_{v}(z)+y_{u}(z+v)$, there exist constant $c_{1}, c_{2}$, and a holomorphic function $h(z)$ such that $y_{1}(z)=c_{2}+h(z+1)-h(z)$, $y_{\tau}(z)=c_{1}+h(z+\tau)-h(z)$. Since $c_{2}(z+1)-c_{2} z=c_{2}$, we may assume $c_{2}=0$.

Applying the observation above to $(\mathrm{d} / \mathrm{d} z) x_{u}$, we have constant $c, \delta$, and a holomorphic function $\phi(z)$ such that $x_{\tau}(z)=c z+\delta+\phi(z+\tau)-\phi(z)$ and $x_{1}(z)=\phi(z+1)-\phi(z)$. The condition (6.1) is equivalent to $c \in L_{\theta}$. Hence $X \simeq X_{c, \delta}$. The homomorphism $\mathrm{H}^{1}(T, \mathcal{O}(E)) \rightarrow \mathrm{H}^{2}(T, H)$ is isomorphic to

$$
\mathrm{H}^{1}\left(L_{\tau}, \mathrm{H}^{0}(\mathbb{C}, \mathcal{O}(E))\right) \rightarrow \mathrm{H}^{2}\left(L_{\tau}, L_{\theta}\right) \simeq L_{\theta},
$$

which sends $\eta$ to $c$. Hence $c \neq 0$.

Definition 6.2. (1) For three complex numbers $x_{1}, x_{2}, x_{3}$, let $T\left(x_{1}, x_{2}, x_{3}\right)$ denote the matrix

$$
\left(\begin{array}{ccc}
1 & 0 & 0 \\
x_{1} & 1 & 0 \\
x_{3} & x_{2} & 1
\end{array}\right) .
$$

The matrices above form a subgroup of $\mathrm{GL}(3, \mathbb{C})$, which is denoted by $T_{3}(\mathbb{C})$. 
(2) Let $D: \mathbb{C}^{2} \times \mathbb{C}^{2} \rightarrow \mathbb{C}$ be the skew symmetric form defined by

$$
D\left(\left(x_{1}, x_{2}\right),\left(x_{1}^{\prime}, x_{2}^{\prime}\right)\right)=x_{1} x_{2}^{\prime}-x_{1}^{\prime} x_{2} .
$$

(3) Let $\Delta_{3}(\mathbb{C})$ be the following group structure on $(\xi, y) \in \mathbb{C}^{2} \times \mathbb{C}$ :

$$
(\xi, y) *\left(\xi^{\prime}, y^{\prime}\right):=\left(\xi+\xi^{\prime}, y+y^{\prime}-(1 / 2) D\left(\xi, \xi^{\prime}\right)\right) .
$$

Note that the $l$-th power $(\xi, y)^{l}$ is equal to $(l \xi, l y)$ for $l \in \mathbb{Z},(\xi, y) \in \Delta_{3}(\mathbb{C})$. There is an isomorphism $T_{3}(\mathbb{C}) \rightarrow \Delta_{3}(\mathbb{C})$ given by

$$
T\left(x_{1}, x_{2}, x_{3}\right) \mapsto\left(\left(x_{1}, x_{2}\right), x_{3}-(1 / 2) x_{1} x_{2}\right) .
$$

We have a homomorphism $\pi_{1}\left(X_{c, \delta}\right) \rightarrow T_{3}(\mathbb{C})$ by

$$
g_{1} \mapsto T(\tau, c, \delta), \quad g_{2} \mapsto T(1,0,0), \quad g_{3} \mapsto T(0,0, \theta), \quad g_{4} \mapsto T(0,0,1) .
$$

Therefore the composite $\pi_{1}\left(X_{c, \delta}\right) \rightarrow \Delta_{3}(\mathbb{C})$ is written by

$$
g_{1}^{l_{1}} g_{2}^{l_{2}} g_{3}^{l_{3}} g_{4}^{l_{4}} \mapsto\left(\left(l_{1} \tau+l_{2}, l_{1} c\right), l_{1} \varepsilon+(1 / 2) l_{1} l_{2} c+l_{3} \theta+l_{4}\right), \quad \text { where } \quad \varepsilon:=\delta-(1 / 2) c \tau .
$$

Definition 6.3. (1) For a free abelian group $L$ of finite rank and for $c \in L$, let $L[c / 2]$ denotes the abelian group $L+\mathbb{Z}(c / 2) \subset L \otimes \mathbb{Q}$.

(2) Let $D_{\tau}: L_{\tau} \times L_{\tau} \rightarrow \mathbb{Z}$ be the skew symmetric form defined by

$$
D_{\tau}\left(m_{1} \tau+m_{2}, m_{1}^{\prime} \tau+m_{2}^{\prime}\right)=m_{1} m_{2}^{\prime}-m_{1}^{\prime} m_{2}
$$

In other expressions,

$$
D_{\tau}(x, y)=\frac{1}{\tau-\bar{\tau}}(x \bar{y}-\bar{x} y)=\frac{\operatorname{Im}(x \bar{y})}{\operatorname{Im} \tau} .
$$

(3) For $c \in L_{\theta}$, let $\Pi_{c}$ be the following group defined on $L_{\tau} \times L_{\theta}[c / 2]$ :

$$
(x, y) *\left(x^{\prime}, y^{\prime}\right):=\left(x+x^{\prime}, y+y^{\prime}+(c / 2) D_{\tau}\left(x, x^{\prime}\right)\right) .
$$

Note that $D_{\tau}(x, 1)=\operatorname{Im} x / \operatorname{Im} \tau$ and $x=D_{\tau}(x, 1) \tau-D_{\tau}(x, \tau)$ for $x \in L_{\tau}$.

We have homomorphisms $\Pi_{c} \rightarrow \Delta_{3}(\mathbb{C})$ and $\pi_{1}\left(X_{c, \delta}\right) \rightarrow \Pi_{c}$, respectively, by

$$
\begin{aligned}
(x, y) & \mapsto\left(\left(x, D_{\tau}(x, 1) c\right), y+D_{\tau}(x, 1) \varepsilon\right), \quad \text { and } \\
g_{1}^{l_{1}} g_{2}^{l_{2}} g_{3}^{l_{3}} g_{4}^{l_{4}} & \mapsto\left(l_{1} \tau+l_{2}, l_{3} \theta+l_{4}+(1 / 2) l_{1} l_{2} c\right) .
\end{aligned}
$$

Then we have the commutative diagram

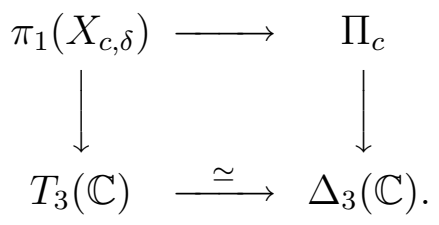

The image of the injection $\pi_{1}\left(X_{c, \delta}\right) \hookrightarrow \Pi_{c}$ consists of all the elements $(x, y)$ such that $y+(c / 2) D_{\tau}(x, 1) D_{\tau}(x, \tau) \in L_{\theta}$. In particular, $\Pi_{c}$ is generated by $\pi_{1}\left(X_{c, \delta}\right)$ and $(0, c / 2)$. The group $\Pi_{c}$ acts on $\mathbb{C} \times \mathbb{C}$ by

$$
(z, \zeta) \mapsto\left(z+x, \zeta+D_{\tau}(x, 1) c z+y+D_{\tau}(x, 1)(\varepsilon+(1 / 2) c x)\right)
$$


for $(x, y) \in \Pi_{c}$.

Proposition 6.4. Let $f: X_{c, \delta} \rightarrow X_{c, \delta}$ be a surjective endomorphism and let $h: T \rightarrow T$ be the induced endomorphism with $\pi \circ f=h \circ \pi$. Suppose that

$$
h_{*}: \mathrm{H}^{0}\left(T, \Theta_{T}\right) \rightarrow \mathrm{H}^{0}\left(T, \Theta_{T}\right)
$$

is the multiplication by $\alpha \in \mathbb{C}$ with $\alpha L_{\tau} \subset L_{\tau}$. Then $f$ is induced from the automorphism

$$
\Phi_{\alpha, v}:(z, \zeta) \mapsto\left(\alpha z+(1 / c)(\alpha-1) \varepsilon,|\alpha|^{2} \zeta+\varphi_{\alpha, v}(z)\right)
$$

of $\mathbb{C} \times \mathbb{C}$ for a holomorphic function

$$
\varphi_{\alpha, v}(z)=\alpha D_{\tau}(\alpha, 1)\left(\frac{c}{2} z^{2}+\varepsilon z\right)+v
$$

for $v \in \mathbb{C}$.

Proof. A lift $\Phi$ of $f$ to $\mathbb{C} \times \mathbb{C}$ is written by $(z, \zeta) \mapsto(\alpha z+\beta, F(z, \zeta))$ for a holomorphic function $F(z, \zeta)$ and for a constant $\beta$. Here, $F(z, \zeta)=\rho \zeta+\varphi(z)$ for a holomorphic function $\varphi(z)$ and a constant $\rho$ since $F(z, \zeta) \bmod L_{\theta}$ depends only on $\zeta \bmod L_{\theta}$. The endomorphism $f_{*}: \pi_{1}\left(X_{c, \delta}\right) \rightarrow \pi_{1}\left(X_{c, \delta}\right)$ is induced from $g \mapsto \Phi \circ g \circ \Phi^{-1}$ and lifts to an endomorphism of $\Pi_{c}$. The image of $(x, y) \in \Pi_{c}$ is $\left(\alpha x, y_{1}\right)$ for some $y_{1} \in L_{\theta}[c / 2]$ in which the following equation holds:

$$
\begin{aligned}
\rho\left(D_{\tau}(x, 1) c z+y+\right. & \left.D_{\tau}(x, 1)(\varepsilon+(1 / 2) c x)\right)+\varphi(z+x) \\
& =\varphi(z)+D_{\tau}(\alpha x, 1) c(\alpha z+\beta)+y_{1}+D_{\tau}(\alpha x, 1)(\varepsilon+(1 / 2) c \alpha x) .
\end{aligned}
$$

By using $\operatorname{Im}(\alpha x)=x \operatorname{Im} \alpha+\bar{\alpha} \operatorname{Im} x$, we have $D_{\tau}(\alpha x, 1)=x D_{\tau}(\alpha, 1)+\bar{\alpha} D_{\tau}(x, 1)$, and

$$
\begin{aligned}
\varphi(z+x)-\varphi(z)= & \left(D_{\tau}(\alpha, 1) \alpha x+D_{\tau}(x, 1)\left(|\alpha|^{2}-\rho\right)\right) c z+y_{1}-\rho y \\
& +(c / 2) D_{\tau}(\alpha, 1) \alpha x^{2}+D_{\tau}(\alpha, 1)(c \beta+\varepsilon) x \\
& +D_{\tau}(x, 1)(\bar{\alpha} c \beta+(\bar{\alpha}-\rho) \varepsilon)+(c x / 2) D_{\tau}(x, 1)\left(|\alpha|^{2}-\rho\right) .
\end{aligned}
$$

Hence $\varphi^{\prime \prime}(z)$ is a constant equal to $D_{\tau}(\alpha, 1) c \alpha$ and $\rho=|\alpha|^{2}$. If we write $\varphi(z)=$ $(c \alpha / 2) D_{\tau}(\alpha, 1) z^{2}+u z+v$ for constants $u, v$, then

$$
u x=D_{\tau}(\alpha, 1)(c \beta+\varepsilon) x+D_{\tau}(x, 1) \bar{\alpha}(c \beta+(1-\alpha) \varepsilon)+y_{1}-|\alpha|^{2} y .
$$

Therefore, $c \beta=(\alpha-1) \varepsilon$ and $u=D_{\tau}(\alpha, 1) \alpha \varepsilon$.

TheOREm 6.5. A primary Kodaira surface and a secondary Kodaira surface admit a non-trivial surjective endomorphism.

Proof. For the primary Kodaira surface $X_{c, \delta}$, the morphism $\Phi_{l, 0}$ for $l>1$ induces a nontrivial surjective endomorphism of degree $l^{6}$. Let $Y$ be a secondary Kodaira surface. Then by Lemma 4.4, there is a cyclic étale covering $X_{c, \delta} \rightarrow Y$ for some $c, \delta$. Then a generator of the cyclic group acts on $X_{c, \delta}$ as $\Phi_{\alpha, v}$ for a root $\alpha$ of unity and for some $v \in \mathbb{C}$. We may assume $\alpha=\exp (2 \pi \sqrt{-1} / k)$, where $k=2,3,4$, or 6 . The order of $\Phi_{\alpha, v}$ is just $k$. If $k>2$, 
then $\tau \in \mathrm{SL}(2, \mathbb{Z}) \alpha$ for the fractionally linear action on $\mathbb{H}$. Hence, we may assume $\alpha=\tau$ if $k>2$. For $l \in \mathbb{Z}$, we define $v[l]$ by $\Phi_{\alpha, v}^{l}=\Phi_{\alpha^{l}, v[l]}$. Then

$$
v[l]=l v+\alpha D_{\tau}(\alpha, 1) \frac{\varepsilon^{2}}{2 c}\left(-l+\sum_{i=0}^{l-1} \alpha^{2 i}\right)
$$

for $l \geq 0$. In fact, it follows from that the term $v_{3}$ in the formula $\Phi_{\alpha_{1}, v_{1}} \circ \Phi_{\alpha_{2}, v_{2}}=\Phi_{\alpha_{1} \alpha_{2}, v_{3}}$ is calculated by

$$
v_{3}=v_{1}+\left|\alpha_{1}\right|^{2} v_{2}+\alpha_{1} D_{\tau}\left(\alpha_{1}, 1\right) \frac{\alpha_{2}^{2}-1}{2 c} \varepsilon^{2} .
$$

If $k=2$, then $v[2]=2 v \in L_{\theta}$. If $k>2$, then $\tau=\alpha$ implies $D_{\tau}(\alpha, 1)=1$ and

$$
v[k]=k\left(v-\frac{\alpha \varepsilon^{2}}{2 c}\right) \in L_{\theta} .
$$

Let $l$ be an integer with $l>1$ and $l^{2} \equiv 1 \bmod k$. Then, for $w_{1}$ and $w_{2}$ defined by $\Phi_{l, 0} \circ \Phi_{\alpha, v}=\Phi_{l \alpha, w_{1}}$ and $\Phi_{\alpha, v} \circ \Phi_{l, 0}=\Phi_{l \alpha, w_{2}}$, we have

$$
w_{2}-w_{1}=\left(v+\alpha D_{\tau}(\alpha, 1) \frac{l^{2}-1}{2 c} \varepsilon^{2}\right)-l^{2} v=-\frac{l^{2}-1}{k} v[k] \in L_{\theta} .
$$

Hence $\Phi_{l, 0}$ induces a non-trivial surjective endomorphism on the quotient space $Y$.

\section{Hopf surfaces}

In this section, we shall prove the following:

THEOREM 7.1. A Hopf surface admits a non-trivial surjective endomorphism if and only if it has at least two elliptic curves.

We set $W$ to be the open set $\mathbb{C}^{2} \backslash\{(0,0)\}$ and $\left(z_{1}, z_{2}\right)$ to be a coordinate system of $\mathbb{C}^{2}$. A Hopf surface is a compact complex surface whose universal covering space is biholomorphic to $W$ by definition. We write the function $\exp (2 \pi \sqrt{-1} z)$ by e $(z)$.

First, we treat the case of elliptic Hopf surfaces with at most two singular fibers.

Let $m_{1}, m_{2}, n$ be positive integers such that $\operatorname{gcd}\left(m_{1}, m_{2}\right)=\operatorname{gcd}\left(n, m_{1}\right)=\operatorname{gcd}\left(n, m_{2}\right)=$ 1 and let $\tau$ be a complex number in $\mathbb{H}$. Let $Y=Y\left(\tau, m_{1}, m_{2}, n\right)$ be the quotient space of $W$ by the following two actions:

$$
A:\left(z_{1}, z_{2}\right) \mapsto\left(\alpha_{1} z_{1}, \alpha_{2} z_{2}\right), \quad B:\left(z_{1}, z_{2}\right) \mapsto\left(\varepsilon_{1} z_{1}, \varepsilon_{2} z_{2}\right)
$$

where $\alpha_{i}=\mathrm{e}\left(m_{i} \tau\right), \varepsilon_{i}=\mathrm{e}\left(m_{i} / n\right)$, for $i=1,2$. Then $Y$ is an elliptic Hopf surface over $\mathbb{P}^{1}$ and smooth over $\mathbb{P}^{1} \backslash\{0, \infty\}$ by the morphism $\left(z_{1}, z_{2}\right) \mapsto\left(z_{1}^{m_{2}}: z_{2}^{m_{1}}\right)$. The multiplicities of the fibers over $0=(1: 0)$ and $\infty=(0: 1)$ are $m_{1}$ and $m_{2}$, respectively. Conversely, we know the following result by Kodaira (cf. Lemma 8 of $[10, \mathrm{II}])$ :

FACT 7.2. Let $Y \rightarrow \mathbb{P}^{1}$ be an elliptic Hopf surface smooth outside $\{0, \infty\}$. Let $m_{1}$ and $m_{2}$ be the multiplicities of the fibers over 0 and $\infty$, respectively. Suppose that $\operatorname{gcd}\left(m_{1}, m_{2}\right)=1$. Then $Y \simeq Y\left(\tau, m_{1}, m_{2}, n\right)$ for some $\tau$ and $n$. 
In particular, if $Y \rightarrow \mathbb{P}^{1}$ is smooth, then $Y \simeq Y(\tau, 1,1, n)$, which is obtained by the actions

$$
A:\left(z_{1}, z_{2}\right) \mapsto \rho\left(z_{1}, z_{2}\right)=\left(\rho z_{1}, \rho z_{2}\right), \quad B:\left(z_{1}, z_{2}\right) \mapsto \mathrm{e}(1 / n)\left(z_{1}, z_{2}\right),
$$

for $\rho=\mathrm{e}(\tau)$. We write $Y(\tau, 1,1, n)$ by $Y(\rho, n)$.

Proposition 7.3. Let $\pi: X \rightarrow T$ be an elliptic Hopf surface with at most two singular fibers. Then $X$ admits a non-trivial surjective endomorphism.

Proof. We may assume that $\pi$ is smooth outside $\{0, \infty\} \subset \mathbb{P}^{1}=T$. Let $m_{1}$ and $m_{2}$ be the multiplicities of the fibers over 0 and $\infty$, respectively. Let $\Gamma=\mathbb{P}^{1} \rightarrow T=\mathbb{P}^{1}$ be the cyclic covering of degree $k=\operatorname{gcd}\left(m_{1}, m_{2}\right)$ branched at $\{0, \infty\}$. Then the normalization $Y$ of $X \times_{T} \Gamma$ is an elliptic Hopf surface étale over $X$. Moreover, $Y \simeq Y\left(\tau, m_{1} / k, m_{2} / k, n\right)$ for some $\tau$ and $n$ by Fact 7.2. A generator of the cyclic Galois group acts on $\Gamma=\mathbb{P}^{1}$ by $\left(t_{1}: t_{2}\right) \mapsto\left(t_{1}: \mathrm{e}(1 / k) t_{2}\right)$. This lifts to an automorphism of $W$ written by

$$
C:\left(z_{1}, z_{2}\right) \mapsto\left(u\left(z_{1}, z_{2}\right)^{m_{1}} z_{1}, u\left(z_{1}, z_{2}\right)^{m_{2}} \mathrm{e}\left(l / m_{1}\right) \mathrm{e}\left(1 / k m_{1}\right) z_{2}\right)
$$

for a unit function $u: \mathbb{C}^{2} \rightarrow \mathbb{C}^{\star}$ and for an integer $l$. We shall show that $u$ is constant. Since it induces an automorphism of $Y$, there is an integer $q$ such that

$$
\begin{aligned}
& u\left(\alpha z_{1}, \alpha z_{2}\right)^{m_{1}} \alpha_{1}=\mathrm{e}\left(m_{1} / n\right)^{q} \alpha_{1}^{ \pm} u\left(z_{1}, z_{2}\right)^{m_{1}}, \\
& u\left(\alpha z_{1}, \alpha z_{2}\right)^{m_{2}} \alpha_{2}=\mathrm{e}\left(m_{2} / n\right)^{q} \alpha_{2}^{ \pm} u\left(z_{1}, z_{2}\right)^{m_{2}},
\end{aligned}
$$

for any $\left(z_{1}, z_{2}\right) \in W$. Substituting $\left(z_{1}, z_{2}\right)=(0,0)$, we have

$$
u\left(\alpha z_{1}, \alpha z_{2}\right)=\mathrm{e}(q / n) u\left(z_{1}, z_{2}\right) .
$$

Then $u$ is constant by

$$
|u(z)|=\lim _{p \rightarrow \infty}\left|u\left(\alpha^{p} z_{1}, \alpha^{p} z_{2}\right)\right|=|u(0,0)| .
$$

Let $\Phi:\left(z_{1}, z_{2}\right) \mapsto\left(z_{1}^{d}, z_{2}^{d}\right)$ be an endomorphism of $W$ for $d>1$. Then $\Phi \circ A=A^{d} \circ \Phi$, $\Phi \circ B=B^{d} \circ \Phi$, and $\Phi \circ C=C^{d} \circ \Phi$. Hence $\Phi$ induces non-trivial surjective endomorphisms on $Y$ and on $X$.

Secondly, we treat the case of elliptic Hopf surfaces with at least three multiple fibers. Let $G \subset \operatorname{PGL}(2, \mathbb{C}) \simeq \operatorname{Aut}\left(\mathbb{P}^{1}\right)$ be a finite subgroup and let $\widetilde{G} \subset \operatorname{SL}(2, \mathbb{C})$ be the pull-back by $\operatorname{SL}(2, \mathbb{C}) \rightarrow \operatorname{PGL}(2, \mathbb{C})$. We denote by $A(g)$ the matrix in $\operatorname{SL}(2, \mathbb{C})$ corresponding to $g \in \widetilde{G}$. We also denote by 1 the unit element of $\widetilde{G}$ and by -1 the element corresponding to the minus of the unit matrix. Note that $G$ is a cyclic group or one of the regular polyhedral groups. We choose $\tau \in \mathbb{H}$ such that $\rho=\mathrm{e}(\tau)$. Let $\chi_{i}: \widetilde{G} \rightarrow \mathbb{C}^{\star}$ be group homomorphisms (characters) for $i=0,1$. Let us choose $\psi_{i}(g) \in \mathbb{Q}$ satisfying $\mathrm{e}\left(\psi_{i}(g)\right)=\chi_{i}(g)$. We define

$$
\varphi(g):=\mathrm{e}\left(\psi_{1}(g) \tau+\psi_{0}(g)(1 / m)\right) .
$$


An action of $\widetilde{G}$ on $Y(\rho, m)$ is well-defined by the maps

$$
\left(z_{1}, z_{2}\right) \mapsto \varphi(g)\left(z_{1}, z_{2}\right)^{\mathrm{t}} A(g)
$$

for $g \in \widetilde{G}$. Thus, an extension $\widetilde{G}_{m, \chi}$ of the finite group $\widetilde{G}$ by $\mathbb{Z} \oplus \mathbb{Z} / m \mathbb{Z}$ acts on $W$. For the action of $g \in \widetilde{G}$ on $Y(\rho, m)$, it has a fixed point if and only if $\varphi(g) \rho^{k} \mathrm{e}(i / m)$ is an eigenvalue of $A(g)$ for some $k$ and $i$. Equivalently, $\chi_{1}(g)=1$ and $\chi_{0}(g)$ is an eigenvalue of $A(g)^{m}$. In particular, $g=-1$ acts trivially on $Y(\rho, m)$ if and only if

$$
\left(\chi_{1}(-1), \chi_{0}(-1)\right)=\left(1,(-1)^{m}\right) \text {. }
$$

We assume this equality holds for $\chi_{1}$ and $\chi_{0}$. Then $G$ acts on $Y(\rho, m)$ and the image $G_{m, \chi}$ of the homomorphism $\widetilde{G}_{m, \chi} \rightarrow \mathrm{GL}(2, \mathbb{C})$ given by the action on $W$ is an extension of $G$ by $\mathbb{Z} \oplus \mathbb{Z} / m \mathbb{Z}$. We also assume that the action of $G$ on $Y(\rho, m)$ is free. This is equivalent to:

$$
\chi_{1}(g) \neq 1 \quad \text { or } \quad \chi_{0}(g) \text { is not an eigenvalue of } A\left(g^{m}\right)
$$

for $g \in \widetilde{G} \backslash\{ \pm 1\}$. Then the quotient space $X(\rho, m, G, \chi):=G \backslash Y(\rho, m)=G_{m, \chi} \backslash W$ is an elliptic Hopf surface over $G \backslash \mathbb{P}^{1}$.

Lemma 7.4. Let $X$ be a Hopf surface with an elliptic fibration $X \rightarrow T$ that has at least three singular fibers. Then $X$ is obtained as the free quotient $X(\rho, m, G, \chi)$ above for some $\rho, m, G, \chi$.

Proof. Let $\pi: X \rightarrow T$ be the elliptic fibration. By the argument in the proof of Lemma 4.4, there is a Galois covering $\tau: \mathbb{P}^{1} \simeq \Gamma \rightarrow T$ such that the normalization $Y$ of $X \times_{T} \Gamma$ is smooth over $\Gamma$ and étale over $X$. Then $Y \simeq Y(\rho, m)$ for some $\rho$ and $m$ by Fact 7.2. The universal covering map $W \rightarrow X$ is the composite of $W \rightarrow Y$ and $Y \rightarrow X$. The action of $G$ on $\Gamma$ lifts to that on $Y$. For $g \in \widetilde{G}$, a lift of the action of $g$ on $Y$ to $W$ is written by

$$
z=\left(z_{1}, z_{2}\right) \mapsto u(z, g) \cdot\left(z_{1}, z_{2}\right)^{\mathrm{t}} A(g)
$$

for a holomorphic function $u: W \times \widetilde{G} \rightarrow \mathbb{C}^{\star}$. The description of the universal covering map $W \rightarrow Y=Y(\rho, m)$ implies that, for $g$, there exist $k, i \in \mathbb{Z}$ such that $u(\rho z, g)=$ $\rho^{k} \mathrm{e}(i / m) u(z, g)$. Since $u$ extends as $\mathbb{C}^{2} \times \widetilde{G} \rightarrow \mathbb{C}^{\star}$, we have $\rho^{k} \mathrm{e}(i / m)=1$ by substituting $z=(0,0)$. Therefore, $u$ descends to $W /\langle\rho\rangle \times \widetilde{G} \rightarrow \mathbb{C}^{\star}$ which is constant by the compactness of the quotient $W /\langle\rho\rangle$. Hence we may write $u(g)=u(z, g) \in \mathbb{C}^{\star}$. Therefore, for any $g_{1}$, $g_{2}$, there exist $k$ and $i$ with $u\left(g_{1} g_{2}\right)=\rho^{k} \mathrm{e}(i / m) u\left(g_{1}\right) u\left(g_{2}\right)$. Hence $u(g)=\varphi(g)$ above for some characters $\chi_{1}$ and $\chi_{0}$. Thus $X$ is isomorphic to the quotient space of $W$ by $G_{m, \chi}$ above and the action of $G_{m, \chi}$ is free since the action of $G$ on $Y$ is free.

LEMMA 7.5. $X(\rho, m, G, \chi)$ admits a non-trivial surjective endomorphism if there exists a $\widetilde{G}$-semi-invariant homogeneous polynomial $H\left(z_{1}, z_{2}\right)$ of degree $d>2$ such that

(1) $H\left(z_{1}, z_{2}\right)$ has only simple zeros over $\mathbb{P}^{1}$, 
(2) $\chi_{1}(g)^{d-2}=\delta(g)^{m} \chi_{0}(g)^{d-2}=1$ for the character $\delta$ determined by

$$
H\left(\left(z_{1}, z_{2}\right)^{\mathrm{t}} A(g)\right)=\delta(g) H\left(z_{1}, z_{2}\right) .
$$

Proof. (cf. [19]) Let $F_{1}\left(z_{1}, z_{2}\right)=-\partial H\left(z_{1}, z_{2}\right) / \partial z_{2}$ and $F_{2}\left(z_{1}, z_{2}\right)=\partial H\left(z_{1}, z_{2}\right) / \partial z_{1}$. Then the morphism $\Phi: W \ni\left(z_{1}, z_{2}\right) \mapsto\left(F_{1}\left(z_{1}, z_{2}\right), F_{2}\left(z_{1}, z_{2}\right)\right) \in W$ is well-defined and

$$
\left(F_{1}\left(\left(z_{1}, z_{2}\right){ }^{\mathrm{t}} A(g)\right), F_{2}\left(\left(z_{1}, z_{2}\right){ }^{\mathrm{t}} A(g)\right)\right)=\delta(g)\left(F_{1}\left(z_{1}, z_{2}\right), F_{2}\left(z_{1}, z_{2}\right)\right){ }^{\mathrm{t}} A(g)
$$

for any $g$. Thus $\Phi$ is $G_{m, \chi}$-equivariant by the condition (2). Hence $\Phi$ induces a nontrivial surjective endomorphism of $X(\rho, m, G, \chi)$ since $\Phi$ induces an endomorphism of $\mathbb{P}^{1}$ of degree $d-1>1$.

Proposition 7.6. The elliptic Hopf surface $X(\rho, m, G, \chi)$ admits non-trivial surjective endomorphisms.

Proof. If $G$ is a cyclic group of order of $n$, then $\widetilde{G}$ is conjugate to the cyclic group generated by

$$
A=\left(\begin{array}{cc}
\mathrm{e}(1 / 2 n) & 0 \\
0 & \mathrm{e}(-1 / 2 n)
\end{array}\right)
$$

in $\operatorname{SL}(2, \mathbb{C})$. Then the elliptic surface $X(\rho, m, G, \chi) \rightarrow G \backslash \mathbb{P}^{1}$ has at most two singular fibers. Hence the existence of non-trivial surjective endomorphisms on $X(\rho, m, G, \chi)$ for a cyclic group $G$ follows from Proposition 7.3.

Thus we assume $G$ is not cyclic. It is enough to construct $H$ satisfying the condition of Lemma 7.5 in the following cases $(2,2, n),(2,3,3),(2,3,4),(2,3,5)$.

Case $(2,2, n): \quad G$ is the dihedral group $D_{n}$ of order $2 n \geq 4$. We may assume $\widetilde{G}$ is generated by

$$
Q:=\sqrt{-1}\left(\begin{array}{ll}
0 & 1 \\
1 & 0
\end{array}\right) \quad \text { and } \quad A:=\left(\begin{array}{cc}
\mathrm{e}(1 / 2 n) & 0 \\
0 & \mathrm{e}(-1 / 2 n)
\end{array}\right)
$$

in $\operatorname{SL}(2, \mathbb{C})$. Then $Q^{2}=A^{n}=-1$ and $Q A Q^{-1}=A^{-1}$. In particular, $A^{2} \in[\widetilde{G}, \widetilde{G}]$. Thus $\widetilde{G} /[\widetilde{G}, \widetilde{G}]$ is isomorphic to $\mathbb{Z} / 4 \mathbb{Z}$ for $n$ odd and to $\mathbb{Z} / 2 \mathbb{Z} \oplus \mathbb{Z} / 2 \mathbb{Z}$ for $n$ even.

If $n$ is even, then $m$ is even by (7.1) since $Q^{2}=-1$.

Let us consider the homogeneous polynomial

$$
H\left(z_{1}, z_{2}\right)=z_{1}^{2 n}-z_{2}^{2 n}
$$

of degree $d=2 n$. This has only simple zeros over $\mathbb{P}^{1}$ and is $\widetilde{G}$-invariant for $n$ odd and $\widetilde{G}$ semi-invariant for $n$ even. Note that $d-2$ is even and moreover $d-2=2(n-1) \equiv 0 \bmod 4$ for $n$ odd. Thus $H$ satisfies the condition of Lemma 7.5 since $\chi_{1}^{d-2}=\chi_{0}^{d-2}=\delta^{m}=1$.

Case $(2,3,3): \quad G$ is the tetrahedral group isomorphic to the alternating group $\mathfrak{A}_{4}$. We may assume that $\widetilde{G}$ is generated by

$$
A=\left(\begin{array}{cc}
\sqrt{-1} & 0 \\
0 & -\sqrt{-1}
\end{array}\right) \quad \text { and } B=\frac{1}{\sqrt{2}} \mathrm{e}(1 / 8)\left(\begin{array}{cc}
1 & \sqrt{-1} \\
1 & -\sqrt{-1}
\end{array}\right)
$$


where we regard $\sqrt{-1}$ as e $(1 / 4)$. Then $A^{2}=B^{3}=-1$ and $(A B)^{3}=1$. Here, $\widetilde{G} /[\widetilde{G}, \widetilde{G}] \simeq$ $\mathbb{Z} / 3 \mathbb{Z}$. In particular, $\chi_{1}^{3}=\chi_{0}^{3}=1$. Let us consider the homogeneous polynomial

$$
H\left(z_{1}, z_{2}\right)=z_{1}^{8}+z_{2}^{8}+14 z_{1}^{4} z_{2}^{4}
$$

of degree $d=8$. Then this has only simple zeros over $\mathbb{P}^{1}$ and is $\widetilde{G}$-invariant. Thus $H$ satisfies the condition of Lemma 7.5 since $d-2 \equiv 0 \bmod 3$.

Case $(2,3,4): \quad G$ is the octahedral group isomorphic to the symmetric group $\mathfrak{S}_{4}$. We may assume that $\widetilde{G}$ is generated by

$$
A=\left(\begin{array}{cc}
\mathrm{e}(1 / 8) & 0 \\
0 & \mathrm{e}(-1 / 8)
\end{array}\right) \quad \text { and } B=\frac{1}{\sqrt{2}} \mathrm{e}(1 / 8)\left(\begin{array}{cc}
1 & \sqrt{-1} \\
1 & -\sqrt{-1}
\end{array}\right) \text {. }
$$

Then $A^{4}=B^{3}=(A B)^{2}=-1$. Here, $\widetilde{G} /[\widetilde{G}, \widetilde{G}] \simeq \mathbb{Z} / 2 \mathbb{Z}$. In particular, the square of any character is trivial. Here $m$ is even by (7.1) since $A^{4}=-1$. Let us consider the homogeneous polynomial

$$
H\left(z_{1}, z_{2}\right)=z_{1} z_{2}\left(z_{1}^{4}-z_{2}^{4}\right)
$$

of degree $d=6$. Then this has only simple zeros over $\mathbb{P}^{1}$ and is $\widetilde{G}$-semi-invariant. Thus $H$ satisfies the condition of Lemma 7.5 since $m$ and $d-2$ are even.

Case $(2,3,5): \quad G$ is the icosahedral group isomorphic to the alternating group $\mathfrak{A}_{5}$. We may assume that $\widetilde{G}$ is generated by

$$
A=-\left(\begin{array}{cc}
\beta^{-2} & 0 \\
0 & \beta^{2}
\end{array}\right) \quad \text { and } \quad B=\frac{1}{\sqrt{5}}\left(\begin{array}{cc}
-\left(\beta-\beta^{-1}\right) & \beta^{2}-\beta^{-2} \\
\beta^{2}-\beta^{-2} & \beta-\beta^{-1}
\end{array}\right)
$$

where $\beta=\mathrm{e}(1 / 5)$. Then $A^{5}=B^{2}=-1$ and $(A B)^{3}=1$. Here, $\widetilde{G}$ has no non-trivial characters. Hence the $\widetilde{G}$-invariant polynomial

$$
H\left(z_{1}, z_{2}\right):=z_{1} z_{2}\left(z_{1}^{10}+11 z_{1} z_{2}-z_{2}^{10}\right)
$$

satisfies the condition of Lemma 7.5.

Finally, we treat the case of non-elliptic Hopf surfaces. By Theorem 32 of [10, II], a non-elliptic Hopf surface $X$ is obtained as the quotient of $W$ by the following action of $\mathbb{Z} \oplus \mathbb{Z} / l \mathbb{Z}$ : A generator of $\mathbb{Z}$ acts as

$$
\left(z_{1}, z_{2}\right) \mapsto\left(\alpha_{1} z_{1}+\lambda z_{2}^{m}, \alpha_{2} z_{2}\right)
$$

where $m$ is a positive integer, $\alpha_{1}, \alpha_{2}, \lambda$ are complex numbers with $0<\left|\alpha_{1}\right| \leq\left|\alpha_{2}\right|<1$ and $\left(\alpha_{1}-\alpha_{2}^{m}\right) \lambda=0$. If $\lambda=0$, then $\alpha_{1}^{p} \neq \alpha_{2}^{q}$ for any positive integers $p, q$; A generator $\mathbb{Z} / l \mathbb{Z}$ acts as

$$
\left(z_{1}, z_{2}\right) \mapsto\left(\varepsilon_{1} z_{1}, \varepsilon_{2} z_{2}\right)
$$

for primitive $l$-the roots $\varepsilon_{1}$, $\varepsilon_{2}$ of unity with $\left(\varepsilon_{1}-\varepsilon_{2}^{m}\right) \lambda=0$. 
The equation $z_{2}=0$ defines an elliptic curve on $X$. If $\lambda \neq 0$, then it is a unique curve of $X$. If $\lambda=0$, then the equation $z_{1}=0$ defines another elliptic curve and there are no other curves contained in $X$.

If $\lambda=0$, then $\left(z_{1}, z_{2}\right) \mapsto\left(z_{1}^{d}, z_{2}^{d}\right)$ for $d>1$ gives a non-trivial surjective endomorphism of $X$. Therefore, the proof of Theorem 7.1 is reduced to the following:

Proposition 7.7. If $\lambda \neq 0$, then $X$ admits no non-trivial surjective endomorphisms.

Proof. We write $\alpha=\alpha_{2}$ and $\varepsilon=\varepsilon_{2}$. Then $\alpha_{1}=\alpha^{m}, \varepsilon_{1}=\varepsilon^{m}$, and $(k, j) \in \mathbb{Z} \oplus \mathbb{Z} / l \mathbb{Z}$ acts on $W$ by

$$
\varphi_{k, j}:\left(z_{1}, z_{2}\right) \mapsto\left(\varepsilon^{j m}\left(\alpha^{k m} z_{1}+k \lambda \alpha^{(k-1) m} z_{2}^{m}\right), \varepsilon^{j} \alpha^{k} z_{2}\right) .
$$

Note that $\varphi_{k, j}$ for $k>0$ is a contraction (cf. Section 10 of [10, II]) in the sense that $\varphi_{k, j}^{n}(B)$ converges to $(0,0)$ for $n \rightarrow+\infty$ for the ball $B=\left\{\left|z_{1}\right|^{2}+\left|z_{2}\right|^{2} \leq 1\right\}$. Suppose that there is an endomorphism $f: X \rightarrow X$. Let $\Phi: W \rightarrow W$ be a lift, which is written by

$$
\Phi:\left(z_{1}, z_{2}\right) \mapsto\left(F\left(z_{1}, z_{2}\right), G\left(z_{1}, z_{2}\right)\right)
$$

for holomorphic functions $F, G$ defined on $\mathbb{C}^{2}$. Here, $\Phi \circ \varphi_{1,0}=\varphi_{p, q} \circ \Phi$ for some integers $p$ and $q$. Hence the following functional equations hold:

$$
\begin{aligned}
& F\left(\alpha^{m} z_{1}+\lambda z_{2}^{m}, \alpha z_{2}\right)=\varepsilon^{q m}\left(\alpha^{p m} F\left(z_{1}, z_{2}\right)+p \lambda \alpha^{(p-1) m} G\left(z_{1}, z_{2}\right)^{m}\right), \\
& G\left(\alpha^{m} z_{1}+\lambda z_{2}^{m}, \alpha z_{2}\right)=\varepsilon^{q} \alpha^{p} G\left(z_{1}, z_{2}\right) .
\end{aligned}
$$

Here, we have $p>0$ by (7.4); otherwise,

$$
\left|G\left(z_{1}, z_{2}\right)\right|=\left|\alpha^{-p k} G\left(\varphi_{k, 0}\left(z_{1}, z_{2}\right)\right)\right| \rightarrow 0 \quad \text { as } \quad k \rightarrow+\infty
$$

for $p<0$ and $G\left(z_{1}, z_{2}\right)$ is constant for $p=0$. Moreover, $F(0,0)=G(0,0)=0$ by

$$
\Phi \circ \varphi_{k, 0}\left(z_{1}, z_{2}\right)=\varphi_{p, q}^{k} \circ \Phi\left(z_{1}, z_{2}\right) \rightarrow \Phi(0,0)=(0,0) \quad \text { as } \quad k \rightarrow+\infty .
$$

We insert here the following:

LEMma 7.8. Let $G\left(z_{1}, z_{2}\right)$ be an entire holomorphic function satisfying (7.4). Then $G\left(z_{1}, z_{2}\right)=c z_{2}^{p}$ for a constant $c$. If $c \neq 0$, then $\varepsilon^{q}=1$.

Proof. We follow the argument of Kodaira in the proof of Theorem 31 of [10, II]. We may assume that $G$ is not identically zero. We set $G_{(\nu)}\left(z_{1}, z_{2}\right):=\partial^{\nu} G\left(z_{1}, z_{2}\right) / \partial z_{1}^{\nu}$ for $\nu \geq 1$. Then

$$
\alpha^{m \nu-p} \varepsilon^{-q} G_{(\nu)}\left(\varphi_{1,0}\left(z_{1}, z_{2}\right)\right)=G_{(\nu)}\left(z_{1}, z_{2}\right)
$$

by (7.4). If $m \nu>p$, then $G_{(\nu)}\left(z_{1}, z_{2}\right) \equiv 0$ by

$$
G_{(\nu)}\left(z_{1}, z_{2}\right)=\alpha^{k(m \nu-p)} \varepsilon^{-q k} G_{(\nu)}\left(\varphi_{k, 0}\left(z_{1}, z_{2}\right)\right) \rightarrow 0 \quad \text { for } \quad k \rightarrow+\infty .
$$

Hence we can write

$$
G\left(z_{1}, z_{2}\right)=\sum_{i=0}^{N} G_{i}\left(z_{2}\right) z_{1}^{i}
$$


for entire holomorphic functions $G_{i}\left(z_{2}\right)$ and for an integer $0 \leq N \leq p / m$ such that $G_{N}$ is not identically zero. By comparing the coefficients of $z_{1}^{i}$ on both sides of (7.4), we have

$$
\varepsilon^{q} \alpha^{p} G_{i}\left(z_{2}\right)=\alpha^{m i} \sum_{l=i}^{N}\left(\begin{array}{l}
l \\
i
\end{array}\right) G_{l}\left(\alpha z_{2}\right) \lambda^{l-i} z_{2}^{m(l-i)},
$$

for $0 \leq i \leq N$. In particular, $G_{N}\left(\alpha z_{2}\right)=\varepsilon^{q} \alpha^{p-m N} G_{N}\left(z_{2}\right)$. Hence $\varepsilon^{q}=1$, and $G_{N}\left(z_{2}\right)=$ $c z_{2}^{p-m N}$ for a constant $c \neq 0$. Suppose that $N \neq 0$. By (7.5) in the case $i=N-1$, we have:

$$
\alpha^{p} G_{N-1}\left(z_{2}\right)=\alpha^{m(N-1)} G_{N-1}\left(\alpha z_{2}\right)+c N \lambda \alpha^{p-m} z_{2}^{p-m N+m} .
$$

By comparing the coefficients of $z_{2}^{k-m N+m}$ on both sides of equation (7.6), we derive a contradiction to $N \neq 0$. Therefore, $N=0$ and $G\left(z_{1}, z_{2}\right)=c z_{2}^{p}$ for some $c \neq 0$.

Proof of Proposition 7.7 continued. We have $\varepsilon^{q}=1$ and $G\left(z_{1}, z_{2}\right)=c z_{2}^{p}$ for a constant $c \neq 0$ by Lemma 7.8. Thus the equation (7.3) is written by

$$
F\left(\alpha^{m} z_{1}+\lambda z_{2}^{m}, \alpha z_{2}\right)=\alpha^{p m} F\left(z_{1}, z_{2}\right)+p \lambda \alpha^{(p-1) m} c^{m} z_{2}^{p m} .
$$

Hence, $F_{(1)}:=\partial F / \partial z_{1}$ satisfies a functional equation

$$
F_{(1)}\left(\alpha^{m} z_{1}+\lambda z_{2}^{m}, \alpha z_{2}\right)=\alpha^{(p-1) m} F_{(1)}\left(z_{1}, z_{2}\right)
$$

similar to (7.4). Thus $F_{(1)}\left(z_{1}, z_{2}\right)=c_{1} z_{2}^{(p-1) m}$ for a constant $c_{1}$ by Lemma 7.8. Then $F\left(z_{1}, z_{2}\right)=c_{1} z_{1} z_{2}^{(p-1) m}+H\left(z_{2}\right)$ for a holomorphic function $H\left(z_{2}\right)$. By $(7.7)$, we have

$$
c_{1} \lambda \alpha^{(p-1) m} z_{2}^{p m}+H\left(\alpha z_{2}\right)=\alpha^{p m} H\left(z_{2}\right)+p \lambda \alpha^{(p-1) m} c^{m} z_{2}^{p m}
$$

and hence $H\left(z_{2}\right)=\delta z_{2}^{p m}$ for a constant $\delta$ and $c_{1}=p c^{m}$. Thus we obtain:

$$
F\left(z_{1}, z_{2}\right)=p c^{m} z_{1} z_{2}^{(p-1) m}+\delta z_{2}^{p m}, \quad G\left(z_{1}, z_{2}\right)=c z_{2}^{p} .
$$

If $p \geq 2$, then $F\left(z_{1}, 0\right) \equiv G\left(z_{1}, 0\right) \equiv 0$, which contradicts the assumption that $F$ and $G$ have no common zeros except $\left(z_{1}, z_{2}\right)=(0,0)$. Hence $p=1$ and

$$
F\left(z_{1}, z_{2}\right)=c^{m} z_{1}+\delta z_{2}^{m}, \quad G\left(z_{1}, z_{2}\right)=c z_{2}
$$

for constants $c \neq 0$ and $\delta$. Thus the endomorphism $f: X \rightarrow X$ is an isomorphism.

\section{InOUe SURFACES Without CURVES}

In the paper [5], Inoue constructed examples of compact complex surface of class $\mathrm{VII}_{0}$ with $b_{2}=0$ having no curves. These are called Inoue surfaces and are denoted by $S_{M}$, $S_{N, p, q, r ; t}^{(+)}$, and $S_{N, p, q, r}^{(-)}$. Moreover Inoue showed in the same paper that if there is an invertible sheaf $\mathcal{L}$ satisfying

$$
\mathrm{H}^{0}\left(S, \Omega_{S}^{1} \otimes \mathcal{L}\right) \neq 0
$$

on a surface $S$ with $b_{1}(S)-1=b_{2}(S)=0$ having no curves, then $S$ is one of the surfaces above. By the works [11], [21], we can remove the assumption on the existence of $\mathcal{L}$ 
above; These Inoue surfaces are characterized as the surfaces with $b_{1}=1, b_{2}=0$ having no curves.

Lemma 8.1. Let $f: X \rightarrow X$ be an étale endomorphism of a surface of class $\mathrm{VII}_{0}$ with $\kappa(X)=-\infty$. Then $f^{*}: \mathrm{H}^{1}(X, \mathbb{Z}) \rightarrow \mathrm{H}^{1}(X, \mathbb{Z})$ is identical.

Proof. Assume the contrary. Then $f^{*}$ is the multiplication map by an integer $d \neq 1$. We have the isomorphism $\mathrm{H}^{1}\left(X, \mathbb{C}^{\star}\right) \simeq \mathrm{H}^{1}\left(X, \mathcal{O}_{X}^{\star}\right)$ from the exponential sequence on $X$. Thus $K_{X} \sim f^{*} K_{X}$ implies that $\mathcal{O}_{X}\left((d-1) m K_{X}\right) \simeq \mathcal{O}_{X}$ for the order $m$ of the torsion part of $\mathrm{H}_{1}(X, \mathbb{Z})$. In particular, $\kappa(X)=0$, a contradiction.

The Inoue surface $S_{M}$ is defined as follows: Let $M$ be a matrix in $\operatorname{SL}(3, \mathbb{Z})$ with eigenvalues $\alpha, \beta, \bar{\beta}$ such that $\alpha>1$ and $\beta \notin \mathbb{R}$. Here, $\alpha \notin \mathbb{Q}$. Let ${ }^{\mathrm{t}}\left(a_{1}, a_{2}, a_{3}\right)$ be a real eigenvector with $\alpha$ as the eigenvalue and let ${ }^{\mathrm{t}}\left(b_{1}, b_{2}, b_{3}\right)$ be an eigenvector with $\beta$ as the eigenvalue. Then three vectors $\left(a_{1}, b_{1}\right),\left(a_{2}, b_{2}\right),\left(a_{3}, b_{3}\right)$ are $\mathbb{R}$-linearly independent and satisfy

$$
\left(\alpha a_{i}, \beta b_{i}\right)=\sum_{j=1}^{3} m_{i j}\left(a_{j}, b_{j}\right), \quad \text { where } \quad M=\left(m_{i j}\right) \in \mathrm{SL}(3, \mathbb{Z}) .
$$

Let $G_{M}$ be the group of automorphisms of $\mathbb{H} \times \mathbb{C}$ generated by

$$
\begin{aligned}
g_{0}:(w, z) & \mapsto(\alpha w, \beta z), \\
g_{i}:(w, z) & \mapsto\left(w+a_{i}, z+b_{i}\right) \text { for } \quad i=1,2,3 .
\end{aligned}
$$

The action of $G_{M}$ on $\mathbb{H} \times \mathbb{C}$ is properly discontinuous and free. The surface $S_{M}$ is defined as the quotient surface of $\mathbb{H} \times \mathbb{C}$ by $G_{M}$. The generators $g_{i}$ satisfy the following relations:

$$
g_{i} g_{j}=g_{j} g_{i}, \quad g_{0} g_{i} g_{0}^{-1}=g_{1}^{m_{i 1}} g_{2}^{m_{i 2}} g_{3}^{m_{i 3}}, \quad \text { for } \quad 1 \leq i, j \leq 3 .
$$

Proposition 8.2. The Inoue surface $S_{M}$ admits a non-trivial surjective endomorphism.

Proof. Let $\Phi$ be the automorphism of $\mathbb{H} \times \mathbb{C}$ given by $(w, z) \mapsto(n w, n z)$ for an integer $n>$ 1. Then $\Phi \circ g_{0}=g_{0} \circ \Phi$ and $\Phi \circ g_{i}=g_{i}^{n} \circ \Phi$ for $1 \leq i \leq 3$. Thus an endomorphism $f: S_{M} \rightarrow$ $S_{M}$ is defined by $\Phi$. Here $f_{*}: \pi_{1}\left(S_{M}\right) \rightarrow \pi_{1}\left(S_{M}\right)$ is isomorphic to the homomorphism $G_{M} \rightarrow G_{M}$ given by $G_{M} \ni g \mapsto \Phi \circ g \circ \Phi^{-1}$. Thus $f$ is non-trivial.

The Inoue surface $S_{N, p, q, r ; t}^{(+)}$is defined for a matrix $N$ in $\operatorname{SL}(2, \mathbb{Z})$ with $n:=\operatorname{tr} N>2$, integers $p, q, r$ with $r \neq 0$, and for a complex number $t$ as follows: Let $\alpha$ be an eigenvalue with $\alpha>1$. Let $\boldsymbol{a}={ }^{\mathrm{t}}\left(a_{1}, a_{2}\right)$ and $\boldsymbol{b}={ }^{\mathrm{t}}\left(b_{1}, b_{2}\right)$ be non-zero real column vectors such that $N \boldsymbol{a}=\alpha \boldsymbol{a}$ and $N \boldsymbol{b}=\alpha^{-1} \boldsymbol{b}$. Note that $a_{i}$ and $b_{i}$ are non-zero and $a_{2} / a_{1}$ and $b_{2} / b_{1}$ are irrational. We set $\theta:=\operatorname{det}(\boldsymbol{a}, \boldsymbol{b})=a_{1} b_{2}-a_{2} b_{1}$. For a pair $\left(l_{1}, l_{2}\right)$ of integers, we set

$$
e\left(l_{1}, l_{2}\right):=\frac{l_{1}\left(l_{1}-1\right)}{2} b_{1} a_{1}+\frac{l_{2}\left(l_{2}-1\right)}{2} b_{2} a_{2}+l_{1} l_{2} b_{1} a_{2} .
$$


We define $e_{1}:=e\left(n_{11}, n_{12}\right)$ and $e_{2}:=e\left(n_{21}, n_{22}\right)$ for the matrix $N=\left(n_{i j}\right)$. We also define a real column vector $\boldsymbol{c}={ }^{\mathrm{t}}\left(c_{1}, c_{2}\right)$ by

$$
(N-I) \boldsymbol{c}+{ }^{\mathrm{t}}\left(e_{1}, e_{2}\right)-(\theta / r)^{\mathrm{t}}(p, q)=0,
$$

where $I$ denotes the unit matrix. Let $G^{(+)}=G_{N, p, q, r ; t}^{(+)}$be the group of automorphisms of $\mathbb{H} \times \mathbb{C}$ generated by

$$
\begin{aligned}
& g_{0}:(w, z) \mapsto(\alpha w, z+t), \\
& g_{i}:(w, z) \mapsto\left(w+a_{i}, z+b_{i} w+c_{i}\right) \text { for } i=1,2, \\
& g_{3}:(w, z) \mapsto(w, z-\theta / r) .
\end{aligned}
$$

Then $g_{3}$ commutes with $g_{i}$ for $0 \leq i \leq 2$. Moreover, we have:

$$
g_{1} g_{2}=g_{2} g_{1} g_{3}^{r}, \quad g_{0} g_{1} g_{0}^{-1}=g_{1}^{n_{11}} g_{2}^{n_{12}} g_{3}^{p}, \quad g_{0} g_{2} g_{0}^{-1}=g_{1}^{n_{21}} g_{2}^{n_{22}} g_{3}^{q} .
$$

These relations determine the group structure of $G^{(+)}$. The subgroup $\Gamma=\Gamma_{r}^{(+)} \subset G^{(+)}$ generated by $g_{1}, g_{2}$, and $g_{3}$ is normal and the quotient $G^{(+)} / \Gamma$ is a free abelian group of rank one generated by the class of $g_{0}$. The center of $G^{(+)}$is generated by $g_{3}$ and contains $[\Gamma, \Gamma]$. The quotient group of $\Gamma$ by the center is a free abelian group of rank two generated by the classes of $g_{1}$ and $g_{2}$. The action of $G^{(+)}$on $\mathbb{H} \times \mathbb{C}$ is properly discontinuous and free. The surface $S_{N, p, q, r ; t}^{(+)}$is defined as the quotient space. More precisely, we denote it by $S_{N, p, q, r ; t}^{(+)}(\boldsymbol{a}, \boldsymbol{b})$.

Definition 8.3. (1) Let $T_{3}$ denote the subgroup of $T_{3}(\mathbb{C})$ consisting of $T\left(x_{1}, x_{2}, x_{3}\right)$ with $x_{i} \in \mathbb{R}$.

(2) Let $\Delta_{3}$ denote the subgroup of $\Delta_{3}(\mathbb{C})$ consisting of $\left(\left(x_{1}, x_{2}\right), y\right)$ with $x_{1}, x_{2}, y \in \mathbb{R}$.

(3) Let $\mathbb{D}: \mathbb{Z}^{2} \times \mathbb{Z}^{2} \rightarrow \mathbb{Z}$ be the skew symmetric form defined by

$$
\mathbb{D}\left(\left(l_{1}, l_{2}\right),\left(l_{1}^{\prime}, l_{2}^{\prime}\right)\right):=l_{1} l_{2}^{\prime}-l_{2} l_{1}^{\prime} .
$$

In other expressions, $\mathbb{D}\left(\xi, \xi^{\prime}\right)=\operatorname{det}\left({ }^{\mathrm{t}} \xi,{ }^{\mathrm{t}} \xi^{\prime}\right)$ for row vectors $\xi, \xi^{\prime} \in \mathbb{Z}^{2}$.

(4) For an integer $r \neq 0$, let $\mathbb{Z}[r / 2]=\mathbb{Z}+\mathbb{Z}(r / 2) \subset \mathbb{Q}$ and let $\Gamma_{r}$ be the following group structure defined on $\mathbb{Z}^{2} \times \mathbb{Z}[r / 2]$ :

$$
(\xi, y) *\left(\xi^{\prime}, y^{\prime}\right):=\left(\xi+\xi^{\prime}, y+y^{\prime}+(r / 2) \mathbb{D}\left(\xi, \xi^{\prime}\right)\right) \text {. }
$$

An element of $\Gamma_{r}$ is denoted by $(\xi, y)$ for a row vector $\xi \in \mathbb{Z}^{2}$ and $y \in \mathbb{Z}[r / 2]$.

The group $T_{3}$ acts on $(w, z) \in \mathbb{H} \times \mathbb{C}$ by the multiplication map

$$
T\left(x_{1}, x_{2}, x_{3}\right)^{\mathrm{t}}(1, w, z)={ }^{\mathrm{t}}\left(1, w+x_{1}, z+x_{2} w+x_{3}\right) .
$$

The group homomorphism $\Gamma=\Gamma_{r}^{(+)} \rightarrow T_{3}$ given by

$$
g_{1} \mapsto T\left(a_{1}, b_{1}, c_{1}\right), \quad g_{2} \mapsto T\left(a_{2}, b_{2}, c_{2}\right), \quad g_{3} \mapsto T(0,0,-\theta / r)
$$


is compatible with the actions on $\mathbb{H} \times \mathbb{C}$. The homomorphism is written explicitly as follows:

$$
g_{1}^{l_{1}} g_{2}^{l_{2}} g_{3}^{l_{3}} \mapsto T\left(\left(l_{1}, l_{2}\right) \boldsymbol{a},\left(l_{1}, l_{2}\right) \boldsymbol{b},\left(l_{1}, l_{2}\right) \boldsymbol{c}-(\theta / r) l_{3}+e\left(l_{1}, l_{2}\right)\right),
$$

where $\left(l_{1}, l_{2}\right) \boldsymbol{a}=l_{1} a_{1}+l_{2} a_{2},\left(l_{1}, l_{2}\right) \boldsymbol{b}=l_{1} b_{1}+l_{2} b_{2}$, and $\left(l_{1}, l_{2}\right) \boldsymbol{c}=l_{1} c_{1}+l_{2} c_{2}$. An isomorphism $T_{3} \rightarrow \Delta_{3}$ is induced from (6.2). There is a homomorphism $\Gamma \rightarrow \Gamma_{r}$ given by

$$
g_{1}^{l_{1}} g_{2}^{l_{2}} g_{3}^{l_{3}} \mapsto\left(\left(l_{1}, l_{2}\right), l_{3}+(r / 2) l_{1} l_{2}\right) .
$$

Then an element $\left(\left(l_{1}, l_{2}\right), \lambda\right) \in \Gamma_{r}$ comes from $\Gamma$ if and only if $\lambda-(r / 2) l_{1} l_{2} \in \mathbb{Z}$. There is also a homomorphism $\Gamma_{r} \rightarrow \Delta_{3}$ given by

$$
(\xi, y) \mapsto\left(\xi(\boldsymbol{a}, \boldsymbol{b}), \xi \boldsymbol{c}^{\prime}-(\theta / r) y\right), \quad \text { where } \quad \boldsymbol{c}^{\prime}:=\boldsymbol{c}-(1 / 2)^{\mathrm{t}}\left(a_{1} b_{1}, a_{2} b_{2}\right) .
$$

Then we infer that the diagram

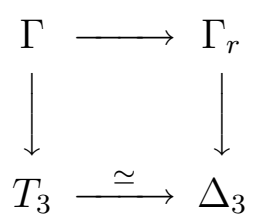

of injective homomorphisms is commutative. The action $g_{0}$ on $\mathbb{H} \times \mathbb{C}$ corresponds to the matrix

$$
A=\left(\begin{array}{lll}
1 & 0 & 0 \\
0 & \alpha & 0 \\
t & 0 & 1
\end{array}\right) .
$$

For the choice of $\boldsymbol{c}$, the relation (8.1) is equivalent to the last two equalities in (8.2). This is also equivalent to

$$
(N-I) \boldsymbol{c}^{\prime}=(\theta / r) \boldsymbol{p}^{\prime}, \quad \text { where } \quad \boldsymbol{p}^{\prime}={ }^{\mathrm{t}}\left(p+(r / 2) n_{11} n_{12}, q+(r / 2) n_{21} n_{22}\right) .
$$

In particular, $G^{(+)}$is a isomorphic to the subgroup of $\mathrm{GL}(3, \mathbb{C})$ generated by the image of $\Gamma \rightarrow \mathrm{GL}(3, \mathbb{R}) \rightarrow \mathrm{GL}(3, \mathbb{C})$ and by the matrix $A$.

Lemma 8.4. (1) An endomorphism $\varphi$ of $\Gamma_{r}$, i.e., a group homomorphism $\varphi: \Gamma_{r} \rightarrow$ $\Gamma_{r}$, is written as

$$
\Gamma_{r} \ni(\xi, y) \mapsto \varphi(\xi, y)=(\xi M, \xi \boldsymbol{v}+(\operatorname{det} M) y)
$$

for a matrix $M \in \mathrm{M}_{2}(\mathbb{Z})$ and a column vector $\boldsymbol{v} \in \mathbb{Z}[r / 2]^{2}$.

(2) The semigroup $\operatorname{End}\left(\Gamma_{r}\right)$ of endomorphisms of $\Gamma_{r}$ is anti-isomorphic to the following semigroup structure on $\mathrm{M}_{2}(\mathbb{Z}) \times \mathbb{Z}[r / 2]^{2}$ :

$$
\left(M_{1}, \boldsymbol{v}_{1}\right) \star\left(M_{2}, \boldsymbol{v}_{2}\right)=\left(M_{1} M_{2}, M_{1} \cdot \boldsymbol{v}_{2}+\left(\operatorname{det} M_{2}\right) \boldsymbol{v}_{1}\right) .
$$

(3) An endomorphism of $\Gamma$ lifts to $\Gamma_{r}$. A pair $(M, \boldsymbol{v}) \in \mathrm{M}_{2}(\mathbb{Z}) \times \mathbb{Z}[r / 2]^{2}$ is induced from an endomorphism of $\Gamma$ if and only if $v_{1}-(r / 2) m_{11} m_{12}, v_{2}-(r / 2) m_{21} m_{22} \in \mathbb{Z}$, where $M=\left(m_{i j}\right), \boldsymbol{v}={ }^{\mathrm{t}}\left(v_{1}, v_{2}\right)$.

(4) The automorphism $\gamma \mapsto g_{0} \gamma g_{0}^{-1}$ of $\Gamma$ corresponds to $\left(N, \boldsymbol{p}^{\prime}\right)$. 
(5) An endomorphism of $G^{(+)}$inducing identity on $G^{(+)} / \Gamma$ is given by an endomorphism $(M, \boldsymbol{v})$ of $\Gamma$ and integers $l_{1}, l_{2}, l_{3}$ satisfying

$$
M N=N M, \quad \text { and } \quad(M-(\operatorname{det} M) I) \boldsymbol{p}^{\prime}-(N-I) \boldsymbol{v}=r M^{\mathrm{t}}\left(l_{2},-l_{1}\right) .
$$

Here, $g_{0}$ is mapped to $g_{0} g_{1}^{l_{1}} g_{2}^{l_{2}} g_{3}^{l_{3}}$.

Proof. For an endomorphism $\varphi$ of $\Gamma_{r}$, we attach $M=\left(m_{i j}\right)$ and $\boldsymbol{v}={ }^{\mathrm{t}}\left(v_{1}, v_{2}\right)$ by

$$
\varphi((1,0), 0)=\left((1,0) M, v_{1}\right) \text { and } \varphi((0,1), 0)=\left((0,1) M, v_{2}\right) .
$$

Then (1) and (2) follow from simple calculations. For (3), it is enough to show that the endomorphism lifts. This is because $\Gamma_{r}$ is generated by $\Gamma$ and an element $((0,0), r / 2)$ commuting with $\Gamma$. (4) follows from the relations (8.2). Let $\rho$ be the endomorphism of (5) and let $\varphi$ be the induced endomorphism of $\Gamma$. Then $\rho\left(g_{0}\right)=g_{0} \eta$ for some $\Gamma \ni \eta=$ $g_{1}^{l_{1}} g_{2}^{l_{2}} g_{3}^{l_{3}}$. Let $\iota(\eta)$ denote the automorphism $\gamma \mapsto \eta \gamma \eta^{-1}$ for $\gamma \in \Gamma$ and let $\nu$ denote another automorphism $\gamma \mapsto g_{0} \gamma g_{0}^{-1}$. Then $\rho$ maps $g_{0} \gamma g_{0}^{-1}=\nu(\gamma)$ to $g_{0} \eta \varphi(\gamma) \eta^{-1} g_{0}^{-1}=\varphi(\nu(\gamma))$. Therefore,

$$
\nu \circ \iota(\eta) \circ \varphi=\varphi \circ \nu .
$$

Conversely, if the relation (8.6) holds, then $\iota(\eta)$ and $\varphi$ define an endomorphism $\rho$ on $G^{(+)}$. Let $(M, \boldsymbol{v}) \in \mathrm{M}_{2}(\mathbb{Z}) \times \mathbb{Z}[r / 2]^{2}$ correspond to $\varphi$. We infer that $\left(I, r^{\mathrm{t}}\left(-l_{2}, l_{1}\right)\right)$ corresponds to $\iota(\eta)$ by $(8.2)$. Thus $(8.6)$ is equivalent to $(8.5)$.

Proposition 8.5. Let $f: X \rightarrow X$ be a surjective endomorphism of the surface $X=$ $S_{N, p, q, r ; t}^{(+)}(\boldsymbol{a}, \boldsymbol{b})$. Then $f$ is induced from the automorphism

$$
\Phi:(w, z) \mapsto\left(c w-\frac{\alpha}{\alpha-1}\left(l_{1}, l_{2}\right) \boldsymbol{a},(\operatorname{det} M) z+\frac{c}{\alpha-1}\left(\left(l_{1}, l_{2}\right) \boldsymbol{b}\right) w+\delta\right),
$$

for a matrix $M \in \mathrm{M}_{2}(\mathbb{Z})$ with a positive eigenvalue $c$, and for integers $l_{1}, l_{2}$, and a complex number $\delta$, in which the following conditions are satisfied:

(1) $\operatorname{det} M \neq 0, M N=N M$, and

$$
(\operatorname{det} M-1) t+\frac{\theta}{2(n-2)}\left(l_{1}, l_{2}\right) N\left(\begin{array}{c}
l_{2} \\
-l_{1}
\end{array}\right)-\left(l_{1}, l_{2}\right) \boldsymbol{c}^{\prime}+(\theta / 2) l_{1} l_{2} \in(\theta / r) \mathbb{Z} .
$$

(2) Let $\boldsymbol{v}={ }^{\mathrm{t}}\left(v_{1}, v_{2}\right)$ be the solution of the equation

$$
(M-(\operatorname{det} M) I) \boldsymbol{p}^{\prime}-(N-I) \boldsymbol{v}=r M\left(\begin{array}{c}
l_{2} \\
-l_{1}
\end{array}\right) .
$$

Then $v_{i}-(r / 2) m_{i 1} m_{12} \in \mathbb{Z}$ for $i=1,2$, where $M=\left(m_{i j}\right)$.

Conversely, if $M$ and $\left(l_{1}, l_{2}\right)$ satisfy the conditions (1), (2), then the automorphism $\Phi$ above induces an endomorphism on $X$ of degree $(\operatorname{det} M)^{2}$. 
Proof. The space $\mathrm{H}^{0}\left(X, \Theta_{X}\right)$ of global holomorphic vector fields on $X$ is one-dimensional and is generated by the vector field $\partial / \partial z$ by Proposition 3 of [5]. Let

$$
\Phi: \mathbb{H} \times \mathbb{C} \ni(w, z) \mapsto\left(\Phi_{1}(w, z), \Phi_{2}(w, z)\right) \in \mathbb{H} \times \mathbb{C}
$$

be a lift of the endomorphism $f$ for some holomorphic functions $\Phi_{i}$. The lift is an automorphism since $f$ is étale. Note that $\Phi_{1}$ depends only on $w$ since any holomorphic mapping $\mathbb{C} \rightarrow \mathbb{H}$ is constant. Thus $\Phi_{1}=F(w)$ for a holomorphic function $F$ on $\mathbb{H}$. The formula $\Phi_{*}(\partial / \partial z)=\partial \Phi_{2} / \partial z(\partial / \partial z)$ implies that $\Phi_{2}=\varepsilon z+G(w)$ for a constant $\varepsilon \neq 0$ and a holomorphic function $G$ on $\mathbb{H}$.

The injective endomorphism $f_{*}: \pi_{1}(X) \rightarrow \pi_{1}(X)$ is given by $\pi_{1}(X) \ni g \mapsto \Phi \circ g \circ \Phi^{-1}$. This defines an element $(M, \boldsymbol{v}) \in \mathrm{M}_{2}(\mathbb{Z}) \times \mathbb{Z}[r / 2]^{2}$ and integers $l_{1}, l_{2}, l_{3}$ by Lemma 8.1 and Lemma 8.4. Here the condition (8.5) is satisfied and $v_{i}-(r / 2) m_{i 1} m_{i 2} \in \mathbb{Z}$ for $i=1,2$, for $M=\left(m_{i j}\right)$ and $\boldsymbol{v}={ }^{\mathrm{t}}\left(v_{1}, v_{2}\right)$. Note that $(\xi, y) \in \Gamma_{r}$ acts on $\in \mathbb{H} \times \mathbb{C}$ by

$$
(w, z) \mapsto\left(w+\xi \boldsymbol{a}, z+(\xi \boldsymbol{b}) w+\xi \boldsymbol{c}^{\prime}-(\theta / r) y+(1 / 2)(\xi \boldsymbol{a})(\xi \boldsymbol{b})\right) .
$$

Hence $\Phi \circ(\xi, y) \circ \Phi^{-1}=(\xi M, \xi \boldsymbol{v}+(\operatorname{det} M) y)$ is equivalent to

$$
\begin{aligned}
& F(w+\xi \boldsymbol{a})=F(w)+\xi M \boldsymbol{a}, \quad \text { and } \\
& \varepsilon\left((\xi \boldsymbol{b}) w+\xi \boldsymbol{c}^{\prime}-(\theta / r) y+(1 / 2)(\xi \boldsymbol{a})(\xi \boldsymbol{b})\right)+G(w+\xi \boldsymbol{a})-G(w) \\
& =(\xi M \boldsymbol{b}) F(w)+\xi M \boldsymbol{c}^{\prime}-(\theta / r)(\xi \boldsymbol{v}+(\operatorname{det} M) y)+(1 / 2)(\xi M \boldsymbol{a})(\xi M \boldsymbol{b}) .
\end{aligned}
$$

Similarly, $\Phi \circ g_{0} \circ \Phi^{-1}=g_{0} g_{1}^{l_{1}} g_{2}^{l_{2}} g_{3}^{l_{3}}$ is equivalent to

$$
\begin{aligned}
& F(\alpha w)=\alpha(F(w)+\zeta \boldsymbol{a}), \quad \text { and } \\
& (\varepsilon-1) t+G(\alpha w)-G(w)=(\zeta \boldsymbol{b}) F(w)+\zeta \boldsymbol{c}^{\prime}-(\theta / r) l^{\prime}+(1 / 2)(\zeta \boldsymbol{a})(\zeta \boldsymbol{b}),
\end{aligned}
$$

where $\zeta=\left(l_{1}, l_{2}\right)$ and $l^{\prime}:=l_{3}+(r / 2) l_{1} l_{2}$. Then $F^{\prime}(w)$ has two periods $a_{1}, a_{2}$ by $(8.7)$. Since $a_{1} / a_{2}$ is irrational, $\mathbb{Z} a_{1}+\mathbb{Z} a_{2} \subset \mathbb{R}$ is dense, which implies that $F^{\prime}(w)$ is constant. Then $G^{\prime \prime}(w)$ has also periods $a_{1}, a_{2}$ by $(8.8)$ and hence $G^{\prime \prime}(w)$ is constant. Moreover $G^{\prime \prime}(w)=0$ by $(8.10)$. We can write

$$
F(w)=c w-\frac{\alpha}{\alpha-1}\left(l_{1}, l_{2}\right) \boldsymbol{a}
$$

for a constant $c$ with $M \boldsymbol{a}=c \boldsymbol{a}$ by (8.7) and (8.9). Note that $c>0$ since $\operatorname{Im} F(w)=$ $c \operatorname{Im} w>0$. Let $c^{\sharp}$ be the conjugate of the algebraic integer $c$ over $\mathbb{Q}$. Then $M \boldsymbol{b}=c^{\sharp} \boldsymbol{b}$. Similarly from (8.10) and (8.8), we have

$$
G(w)=\frac{c}{\alpha-1}\left(\left(l_{1}, l_{2}\right) \boldsymbol{b}\right) w+\delta, \quad c M \boldsymbol{b}=\varepsilon \boldsymbol{b}
$$


for some $\delta \in \mathbb{C}$. Thus $\varepsilon=c c^{\sharp}=\operatorname{det} M$. We note that

$$
\begin{gathered}
\theta=\frac{a_{2} b_{2}}{n_{21}}\left(\alpha-\alpha^{-1}\right), \quad 1 / 2-\alpha /(\alpha-1)=-\frac{\alpha+1}{2(\alpha-1)}=-\frac{1}{2(n-2)}\left(\alpha-\alpha^{-1}\right), \\
\left(\left(l_{1}, l_{2}\right) \boldsymbol{a}\right)\left(\left(l_{1}, l_{2}\right) \boldsymbol{b}\right)=\frac{a_{2} b_{2}}{n_{21}}\left(l_{1}, l_{2}\right) N\left(\begin{array}{c}
l_{2} \\
-l_{1}
\end{array}\right) .
\end{gathered}
$$

Thus (8.10) is written by

$$
\begin{aligned}
(\operatorname{det} M-1) t & =(1 / 2-\alpha /(\alpha-1))(\zeta \boldsymbol{a})(\zeta \boldsymbol{b})+\zeta \boldsymbol{c}^{\prime}-(\theta / r) l^{\prime} \\
& =-\frac{\theta}{2(n-2)}\left(l_{1}, l_{2}\right) N\left(\begin{array}{c}
l_{2} \\
-l_{1}
\end{array}\right)-\left(l_{1}, l_{2}\right) \boldsymbol{c}^{\prime}-(\theta / 2) l_{1} l_{2}-(\theta / r) l_{3} .
\end{aligned}
$$

Hence the conditions (1) and (2) required for $M$ and $\left(l_{1}, l_{2}\right)$ are satisfied. Conversely, suppose that the conditions are satisfied. The condition (8.8) for any $(\xi, y) \in \Gamma_{r}$ is equivalent to

$$
(M-(\operatorname{det} M) I) \boldsymbol{c}^{\prime}=\frac{c}{\alpha-1}\left(\left(l_{1}, l_{2}\right) \boldsymbol{b}\right) \boldsymbol{a}+\frac{\alpha c^{\sharp}}{\alpha-1}\left(\left(l_{1}, l_{2}\right) \boldsymbol{a}\right) \boldsymbol{b}+(\theta / r) \boldsymbol{v} .
$$

By (8.4) and (8.5), it is also equivalent to

$$
\theta M\left(\begin{array}{c}
l_{2} \\
-l_{1}
\end{array}\right)=c\left(\left(l_{1}, l_{2}\right) \boldsymbol{b}\right) \boldsymbol{a}-c^{\sharp}\left(\left(l_{1}, l_{2}\right) \boldsymbol{a}\right) \boldsymbol{b} .
$$

In other words, $Z^{\mathrm{t}}\left(l_{2},-l_{1}\right)=0$ for the matrix

$$
Z:=\theta M-c \boldsymbol{a}\left(b_{2},-b_{1}\right)+c^{\sharp} \boldsymbol{b}\left(a_{2},-a_{1}\right) .
$$

However, $Z \boldsymbol{a}=Z \boldsymbol{b}=0$ by a direct calculation. Hence $Z=0$. Therefore, $\Phi \pi_{1}(X) \Phi^{-1} \subset$ $\pi_{1}(X)$ for the automorphism $\Phi$. Thus an endomorphism of $X$ is induced.

THEOREM 8.6. $S_{N, p, q, r ; t}^{(+)}(\boldsymbol{a}, \boldsymbol{b})$ admits a non-trivial surjective endomorphism if and only if $t \in \mathbb{Q} \theta$, where $\theta=\operatorname{det}(\boldsymbol{a}, \boldsymbol{b})$.

Proof. If the endomorphism exists, then $t \in \mathbb{Q} \theta$ by Proposition 8.5-(1). Conversely suppose that $t \in \mathbb{Q} \theta$. We consider a matrix $M=\left(m_{i j}\right)=k N+I$ for an even integer $k>0$. Then $M$ has a positive eigenvalue $c=k \alpha+1$ and $\operatorname{det} M=k^{2}+k n+1>1$. It is enough to show that, $M$ satisfies the conditions (1) and (2) of Proposition 8.5 for $\left(l_{1}, l_{2}\right)=(0,0)$ for some $k>0$. By assumption,

$$
(\operatorname{det} M-1) t=k(k+n) t \in \mathbb{Z}(\theta / r)
$$

for some $k$. Let $\boldsymbol{v}$ be the solution of $(M-(\operatorname{det} M) I) \boldsymbol{p}^{\prime}=(N-I) \boldsymbol{v}$. Since $m_{11} m_{12}=$ $k\left(k n_{11}+1\right) n_{12}$ and $m_{21} m_{22}=k n_{21}\left(k n_{22}+1\right)$ are even, we have only to show that $\boldsymbol{v} \in \mathbb{Z}^{2}$. We note that $(N-I)^{-1}=(2-n)^{-1}\left(N^{-1}-I\right)$ and $M-(\operatorname{det} M) I=k(N-(k+n) I)$. Thus if $k$ is divisible by $n-2$, then $\boldsymbol{v} \in \mathbb{Z}^{2}$. 
The Inoue surface $S_{M, p, q, r}^{(-)}$is defined in $[5, \S 4]$ for a matrix $M \in \mathrm{M}_{2}(\mathbb{Z})$ with $\operatorname{det} M=-1$, $\operatorname{tr} M>0$ and for integers $p, q, r \neq 0$. The surface $S^{(-)}=S_{M, p, q, r}^{(-)}$has an Inoue surface $S^{(+)}=S_{N, p_{1}, q_{1}, r ; 0}^{(+)}$as an unramified double covering for $N=M^{2}$ and for suitable integers $p_{1}, q_{1}$. The involution of $S^{(+)}$generating the Galois group is induced from $\Psi:(w, z) \mapsto$ $(\beta w,-z)$ for the positive eigenvalue $\sqrt{\alpha}=\beta$ of $M$.

THEOREM 8.7. $S_{M, p, q, r}^{(-)}$admits a non-trivial surjective endomorphism.

Proof. We consider an endomorphism of $S^{(+)}$given by

$$
\Phi:(w, z) \mapsto\left((k \alpha+1) w,\left(k^{2}+k n+1\right) z\right)
$$

for a suitable integer $k>0$ as in Theorem 8.6. Then $\Psi \circ \Phi=\Phi \circ \Psi$. Thus $\Phi$ also gives a non-trivial surjective endomorphism of $S^{(-)}$.

\section{INOUE SURFACES WITH CURVES}

A parabolic Inoue surface, a hyperbolic Inoue surface, and a half Inoue surface are the first examples of surfaces $X$ of class $\mathrm{VII}_{0}$ with $a(X)=0, b_{2}(X)>0$. Different descriptions from [7] of these surfaces are given in [20] by the theory of torus embeddings.

A parabolic Inoue surface $X_{\lambda, n}$ for a complex number $\lambda$ with $0<|\lambda|<1$ and for a positive integer $n$ is given as the quotient space of a toric variety $\mathbb{T}_{N}(\Sigma)$ by an automorphism $g_{\lambda}^{n}$ of infinite order which are defined as follows: $\mathrm{N}$ is a free abelian group of rank two with basis $e_{1}, e_{2}$ and the fan $\Sigma$ consists of the cones

$$
\{0\}, \quad \mathbb{R}_{\geq 0} e_{2}, \quad \mathbb{R}_{\geq 0}\left(e_{1}+\nu e_{2}\right), \quad \mathbb{R}_{\geq 0}\left(e_{1}+\nu e_{2}\right)+\mathbb{R}_{\geq 0}\left(e_{1}+(\nu-1) e_{2}\right)
$$

for all $\nu \in \mathbb{Z}$. Let $g_{\lambda}$ be the automorphism of the open orbit $\mathbb{T}_{N}=N \otimes \mathbb{C}^{\star}$ given by

$$
\left(z, z^{\prime}\right) \mapsto\left(\lambda z, z z^{\prime}\right)
$$

where $\left(z, z^{\prime}\right) \in\left(\mathbb{C}^{\star}\right)^{2}$ corresponds to $z \otimes e_{1}+z^{\prime} \otimes e_{2}$. Then $g_{\lambda}$ extends holomorphically to an automorphism of $\mathbb{T}_{\mathrm{N}}(\Sigma)$. Note that $g_{\lambda}^{n}$ is given by

$$
\left(z, z^{\prime}\right) \mapsto\left(\lambda^{n} z, \lambda^{\frac{n(n-1)}{2}} z^{n} z^{\prime}\right) .
$$

The surface $X_{\lambda, n}$ is of class $\mathrm{VII}_{0}$ with $b_{2}(X)=n$. It contains an elliptic curve $E$ with $E^{2}=-n$ and a cycle $D$ of rational curves consisting of $n$ irreducible components with $D^{2}=0$. Here, $E$ is the quotient curve of the orbit corresponding to $\mathbb{R}_{\geq 0} e_{2}$ and an irreducible component of $D$ is the quotient of the orbit corresponding to $\mathbb{R}_{\geq 0}\left(e_{1}+\nu e_{2}\right)$ for some $\nu$.

Proposition 9.1. Parabolic Inoue surfaces $X_{\lambda, n}$ admit non-trivial surjective endomorphisms. 
Proof. For an integer $k>1$, let $h_{k}$ be the following endomorphism of $\mathbb{T}_{\mathrm{N}}$ :

$$
\left(z, z^{\prime}\right) \mapsto\left(z^{k}, z^{\frac{k(k-1)}{2}} z^{\prime k^{2}}\right) .
$$

Then $h_{k}$ extends to an endomorphism of $\mathbb{T}_{\mathrm{N}}(\Sigma)$ and $g_{\lambda}^{k} \circ h_{k}=h_{k} \circ g_{\lambda}$. Thus $h_{k}$ induces a non-trivial surjective endomorphism on $X_{\lambda, n}$.

A hyperbolic Inoue surface $X_{\mathfrak{K}, \mathrm{N}}$ and a half Inoue surface $\widehat{X}_{\mathfrak{K}, \mathrm{N}}$ are defined as follows for a real quadratic field $\mathfrak{K}$ and for a free abelian subgroup $N \subset \mathfrak{K}$ of rank two generating $\mathfrak{K}$ over $\mathbb{Q}$ : Let $\mathfrak{K} \otimes_{\mathbb{Q}} \mathbb{R} \rightarrow \mathbb{R}^{2}$ be the isomorphism given by $\xi \mapsto\left(\xi, \xi^{\sharp}\right)$ for $\xi \in \mathfrak{K}$ and for the conjugate $\xi^{\sharp}$ over $\mathbb{Q}$. We set

$$
\Gamma_{\mathrm{N}}=\left\{u \in \mathcal{O}_{\mathfrak{K}}^{\times} \mid u>0, u \mathrm{~N}=\mathrm{N}\right\} \quad \text { and } \quad \Gamma_{\mathrm{N}}^{+}=\left\{u \in \Gamma_{\mathrm{N}} \mid u^{\sharp}>0\right\},
$$

where $\mathcal{O}_{\mathfrak{K}}^{\times}$is the unit group of the $\operatorname{ring} \mathcal{O}_{\mathfrak{K}}$ of integers of $\mathfrak{K}$. Then $\Gamma_{N} \simeq \mathbb{Z}$ and $\Gamma_{N}^{+}$is a subgroup of index at most two. Let $\Theta_{N}$ and $\Theta_{N}^{\prime}$ be the convex hulls of $N \cap\left(\mathbb{R}_{>0} \times \mathbb{R}_{>0}\right)$ and $N \cap\left(\mathbb{R}_{>0} \times \mathbb{R}_{<0}\right)$, respectively. Let $\Sigma_{N}$ be the fan of $N \otimes \mathbb{R}=\mathbb{R}^{2}$ corresponding to the decomposition of $\mathbb{R}_{>0} \times(\mathbb{R} \backslash\{0\})$ into sectors by rays joining 0 and a point of

$$
\mathrm{N} \cap\left(\partial \Theta_{\mathrm{N}} \cup \partial \Theta_{\mathrm{N}}^{\prime}\right)
$$

Then $\Gamma_{\mathrm{N}}^{+}$acts on the toric variety $\mathbb{T}_{\mathrm{N}}(\Sigma)$ by $u \times: \mathrm{N} \rightarrow \mathrm{N}$. If $\Gamma_{\mathrm{N}}^{+}$is of index two in $\Gamma_{\mathrm{N}}$, then $\Gamma_{N}$ also acts on the toric variety. Let $\operatorname{Mc}_{N}(\Sigma)$ be the topological quotient space of $\mathbb{T}_{\mathrm{N}}(\Sigma)$ by the compact torus $\mathrm{N} \otimes \mathrm{U}(1) \subset \mathbb{T}_{\mathrm{N}}=\mathrm{N} \otimes \mathbb{C}^{\star}$, where $\mathrm{U}(1)=\{z \in \mathbb{C}|| z \mid=1\}$. Let $\operatorname{ord}_{N}: \mathbb{T}_{N}(\Sigma) \rightarrow \operatorname{Mc}_{N}(\Sigma)$ be the quotient map. Its restriction to $\mathbb{T}_{N}$ is described as the composite

$$
\operatorname{ord}_{N}: N \otimes \mathbb{C}^{\star} \stackrel{\mathrm{id} \otimes|\cdot|}{\longrightarrow} N \otimes \mathbb{R}_{>0} \stackrel{\mathrm{id} \otimes(-\log )}{\simeq} \mathrm{N} \otimes \mathbb{R}
$$

in which the first arrow is induced from the norm map $z \mapsto|z|$ and the second from $0<r \mapsto-\log r$. Let $V_{\mathrm{N}}$ be the pull-back by $\operatorname{ord}_{\mathrm{N}}$ of the open subset

$$
\left(\mathbb{R}_{>0} \times \mathbb{R}\right) \cup\left(\operatorname{Mc}_{N}(\Sigma) \backslash \mathrm{N} \otimes \mathbb{R}\right) .
$$

Then the hyperbolic Inoue surface $X_{\mathfrak{K}, \mathrm{N}}$ is defined as the quotient space of $V_{\mathrm{N}}$ by the action of $\Gamma_{N}^{+}$. The half Inoue surface $\widehat{X}_{\mathfrak{K}, \mathrm{N}}$ is defined in the case $\left[\Gamma_{\mathrm{N}}: \Gamma_{\mathrm{N}}^{+}\right]=2$ as the quotient space $V_{\mathrm{N}}$ by the action of $\Gamma_{\mathrm{N}}$.

Proposition 9.2. Hyperbolic Inoue surfaces and half Inoue surfaces admit non-trivial surjective endomorphisms.

Proof. For a positive integer $l>1$, the multiplication $\mathrm{N} \rightarrow \mathrm{N}$ by $l$ defines an endomorphism of $\mathbb{T}_{\mathrm{N}}(\Sigma)$ of degree $l^{2}>1$. This preserves $V_{\mathrm{N}}$ and commutes with the action of $\Gamma_{\mathrm{N}}^{(+)}$or $\Gamma_{\mathrm{N}}$. Thus a non-trivial surjective endomorphism of degree $l^{2}$ is induced.

Corollary 9.3. Let $X$ be a successive blowups of an Inoue surface with curves whose centers are nodes of curves. Then $X$ admits a non-trivial surjective endomorphisms. 
Proof. Let $Y$ be an Inoue surface with curves and let $f: Y \rightarrow Y$ be a non-trivial surjective endomorphism. By replacing $f$ by some power $f^{k}$, if necessary, we may assume that $f^{-1}(C)=C$ for any curve $C$. Then $f^{-1}(P)=P$ for any node of the union $\cup C$ of all curves. Let $Y_{1} \rightarrow Y$ be the blowup at a node $P$. Then $f$ induces a non-trivial surjective endomorphism $f_{1}: Y_{1} \rightarrow Y_{1}$ which also preserves any curve on $Y_{1}$. In particular, $f_{1}^{-1}\left(P_{1}\right)=P_{1}$ for any node $P_{1}$ of the union of all the curves of $Y_{1}$. Therefore, if $X \rightarrow Y$ is a succession of blowups whose centers are nodes of curves, then a non-trivial surjective endomorphism on $X$ is induced from $f$.

\section{REFERENCES}

[1] W. Barth, C. Peters and A. Van de Ven, Compact complex surfaces, Ergeb. Math. Grenzgeb. (3) 4, Springer-Verlag, Berlin, 1984.

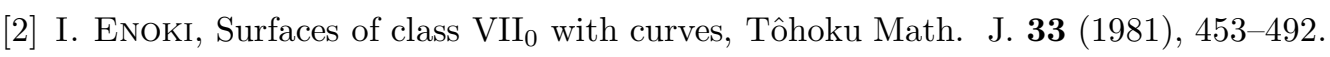

[3] Y. Fujimoto, Endomorphisms of smooth projective 3-folds with non-negative Kodaira dimension, Publ. RIMS. Kyoto Univ. 38 (2002), 33-92.

[4] Y. Fujimoto and E. Sato, On smooth projective threefolds with non-trivial surjective endomorphisms, Proc. Japan Acad. 74 (1998), 143-145.

[5] M. Inoue, On surfaces of class VII ${ }_{0}$, Invent. Math. 24 (1974), 269-310.

[6] M. Inoue, New surfaces with no meromorphic functions, Proc. Int. Cong. Math. (Vancouver, 1974), 423-426, Canad. Math. Congress, Montreal, 1975.

[7] M. Inoue, New surfaces with no meromorphic functions II, Complex Analysis and Algebraic Geometry, A collection of papers dedicated to K. Kodaira (Eds. W. L. Baily, Jr. and T. Shioda), 91-106, Iwanami Shoten Publishers, Tokyo and Cambridge Univ. Press, Cambridge, 1977.

[8] Ma. Kato, Compact complex manifolds containing global spherical shells, I, Proc. Internat. Sympo. Algebraic Geometry, Kyoto, 1997, (Ed. M. Nagata), 45-84, Kinokuniya, Tokyo, 1978.

[9] K. Kodaira, On compact complex analytic surfaces, I-III, Ann. of Math. 71 (1960), 111-152; 77 (1963), 563-626; 78 (1963), 1-40.

[10] K. Kodaira, On the structure of compact complex analytic surfaces, I-IV, Amer. J. Math., 86 (1964), 751-798; 88 (1966), 682-721; 90 (1968), 55-83; 90 (1968), 170-192.

[11] J. LI, S. T. YAU AND F. ZhenG, A simple proof of Bogomolov's theorem on class $\mathrm{VII}_{0}$ surfaces with $b_{2}=0$, Illinois J. Math. 34 (1990), 217-220.

[12] Y. MiYaOKA, Kähler metrics on elliptic surfaces, Proc. Japan Acad. 50 (1974), 533-536.

[13] I. Nakamura, On surfaces of class VII ${ }_{0}$ with curves, Invent. Math. 78 (1984), 393-443.

[14] I. Nakamura, On surfaces of class VII 0 with curves, II, Tôhoku Math. J. 42 (1990), 475-516.

[15] N. Nakayama, On Weierstrass models, Algebraic Geometry and Commutative Algebra. vol. II, In honor of M. Nagata(Eds. H. Hijikata et al.), 405-431, Kinokuniya, Tokyo, 1988.

[16] N. NAKAYAma, Projective algebraic varieties whose universal covering spaces are biholomorphic to $\mathbb{C}^{n}$, J. Math. Soc. Japan 51 (1999), 643-654.

[17] N. Nakayama, Local structure of an elliptic fibration, Higher Dimensional Birational Geometry (Eds. Y. Miyaoka and S. Mori), Adv. Studies in Pure Math. 35, 185-295, Math. Soc. Japan, 2002.

[18] N. Nakayama, Global structure of an elliptic fibration, Publ. RIMS Kyoto Univ. 38 (2002), 451649.

[19] N. Nakayama, Ruled surfaces with non-trivial surjective endomorphisms, Kyushu J. Math. 56 (2002), 433-446. 
[20] T. OdA, Torus embeddings and applications, Based on joint work with K. Miyake, Tata Inst. Fund. Res. 58, Springer-Verlag, Berlin-New York, 1978.

[21] A. Teleman, Projectively flat surfaces and Bogomolov's theorem on class $\mathrm{VII}_{0}$ surfaces, Intern. J. Math. 5 (1994), 253-264.

Department of Mathematics, Faculty of Education

Gifu University, Gifu 501-1193

JAPAN

E-mail address: fujimoto@cc.gifu-u.ac.jp

Research Institute for Mathematical Sciences

Kyoto University, Куото 606-8502

JAPAN

E-mail address: nakayama@kurims.kyoto-u.ac.jp 